\title{
DevKidCC allows for robust classification and direct comparisons of kidney organoid datasets
}

Sean B. Wilson 1,2, Sara E. Howden 1,2, Jessica M. Vanslambrouck', Aude Dorison', Jose Alquicira-Hernandez ${ }^{3}$, Joseph E. Powell ${ }^{3,4}$ and Melissa H. Little $1,2,5,6^{*}$ (D)

\begin{abstract}
Background: While single-cell transcriptional profiling has greatly increased our capacity to interrogate biology, accurate cell classification within and between datasets is a key challenge. This is particularly so in pluripotent stem cell-derived organoids which represent a model of a developmental system. Here, clustering algorithms and selected marker genes can fail to accurately classify cellular identity while variation in analyses makes it difficult to meaningfully compare datasets. Kidney organoids provide a valuable resource to understand kidney development and disease. However, direct comparison of relative cellular composition between protocols has proved challenging. Hence, an unbiased approach for classifying cell identity is required.

Methods: The R package, scPred, was trained on multiple single cell RNA-seq datasets of human fetal kidney. A hierarchical model classified cellular subtypes into nephron, stroma and ureteric epithelial elements. This model, provided in the R package DevKidCC (github.com/KidneyRegeneration/DevKidCC), was then used to predict relative cell identity within published kidney organoid datasets generated using distinct cell lines and differentiation protocols, interrogating the impact of such variations. The package contains custom functions for the display of differential gene expression within cellular subtypes.
\end{abstract}

Results: DevKidCC was used to directly compare between distinct kidney organoid protocols, identifying differences in relative proportions of cell types at all hierarchical levels of the model and highlighting variations in stromal and unassigned cell types, nephron progenitor prevalence and relative maturation of individual epithelial segments. Of note, DevKidCC was able to distinguish distal nephron from ureteric epithelium, cell types with overlapping profiles that have previously confounded analyses. When applied to a variation in protocol via the addition of retinoic acid, DevKidCC identified a consequential depletion of nephron progenitors.

Conclusions: The application of DevKidCC to kidney organoids reproducibly classifies component cellular identity within distinct single-cell datasets. The application of the tool is summarised in an interactive Shiny application, as are examples of the utility of in-built functions for data presentation. This tool will enable the consistent and rapid comparison of kidney organoid protocols, driving improvements in patterning to kidney endpoints and validating new approaches.

Keywords: Cell identity prediction, Human developing kidney, Kidney organoid

\footnotetext{
${ }^{*}$ Correspondence: melissa.little@mcri.edu.au

${ }^{5}$ Department of Anatomy and Neuroscience, The University

of Melbourne, Victoria, Parkville, Australia

Full list of author information is available at the end of the article
} 


\section{Background}

Single-cell RNA sequencing has transformed how we approach biological questions at the transcriptional level, facilitating accurate evaluation of cellular heterogeneity within complex samples, including entire tissues. When coupled with approaches for molecular lineage tagging [1] and computational approaches to analyse pseudotime [2-4] and RNA velocity [5, 6], gene expression in complex tissues such as the kidney can be studied at an unprecedented resolution. Despite these advantages, classification of cellular identity remains challenging and variable between datasets, even when analysing similar cellular systems. Currently, a common approach for identifying cell populations within single-cell data is to first cluster cells, compute differentially expressed genes between clusters and label clusters of cells based on expression of known marker genes $[4,7,8]$. The choice of clusters can be arbitrary, with users defining the number of clusters, thereby raising the potential for biases in the reproducibility of cell-type labels [9]. Placement of cells into a cluster relies on transcriptional similarity [10], hence there needs to be a large enough population with a distinct gene signature for this to occur. Cell clusters are also commonly defined based upon one or a few known differentially expressed genes rather than their global transcriptional signature. Finally, technical challenges such as batch variation can impact definitive cellular identification.

The application of single-cell profiling to developmental biology presents unique challenges due to the presence of intermediate cell types undergoing differentiation during morphogenesis. The mammalian kidney contains more than 25 cell types in the mature postnatal tissue, arising from a smaller number of progenitor cell types including nephron, stromal, endothelial and ureteric progenitors. Organogenesis is driven via reciprocal signalling and self-organisation with many intermediate transcriptional states that are less well defined, making the classification of cell types at the single-cell level both extremely useful but particularly difficult (reviewed in Little and Combes [11]). This is further complicated with hPSC-derived kidney organoid datasets. While protocols for differentiating kidney organoids from hPSC attempt to replicate in vivo kidney differentiation, they are limited and contain emerging non-specific, off-target, or synthetic cell types [12-15]. Here, unbiased classification of cellular identity is a computational challenge. Indeed, recent single-cell profiling of human fetal kidney (HFK) datasets have shown that the classical canonical markers for many cell identities within the kidney are not unique to these cell types but are also expressed at lower levels within other populations [15-18]. This makes cell classification in organoids more challenging when analysing gene expression of these markers in the single-cell clusters. The ability to robustly identify and classify cells in hPSC-derived organoid data is crucial to facilitate useful comparisons between datasets, particularly data generated using different differentiation protocols and cell lines as well as in response to mutation or perturbation. To compare between organoid protocols, studies have generated organoids for data integration and direct comparison [12, 14]. In other work, existing data has been integrated with new data with batch correction methods $[19,20]$ to identify conserved and unique features. These analyses help to improve and refine protocols towards a more accurate endpoint tissue.

One approach to cellular identification is to apply a small set of 'known' genes to identify clusters within a dataset based upon an existing reference dataset that has been accurately classified. Reviewing 13 published kidney or ureteric bud organoid single-cell RNA-seq datasets (Table 1), seven used a published HFK reference to find congruence with their clustered organoid cell populations either through integration or training a unique random forest classifier. However, many different HFK references were used across these publications while other analyses simply selected DE genes for classification without a reference source. Cell classification may be inconsistent when using various references containing different proportions of cells, possibly captured at different ages or regions of the tissue. Indeed, the most commonly used HFK reference only contained cells from the cortex of a 16-week kidney and hence was reported to contain few nephron cells and no ureteric epithelium [27]. There have been many tools developed to utilise reference data to classify a related query dataset, with scrna-tools.org [4] listing 110 tools in the 'Classification' category. These tools extract cell type information from an annotated reference and apply that to a query dataset. Most rely upon the user to supply the reference data and for those that supply a reference, none are directly relevant to hPSC-derived kidney organoids. The $R$ packages scTyper [50] and scClassify [51] have models pre-trained on available kidney references; however, these are not ideal for the classification of the human developing kidney, due to training on mouse cell data (scClassify), or using gene sets of limited adult kidney cell types rather than developing kidney cell populations (scTyper). The browser-based tool, Azimuth, from HuBMAP (https:// azimuth.hubmapconsortium.org/) [52] provides reference-based mapping for an uploaded single-cell gene expression matrix; however, the relevant available references are either human adult kidney or a human fetal development, the latter lacking the required granularity for the developing kidney. As such, there is a need for a tool that can be used to directly and accurately classify 


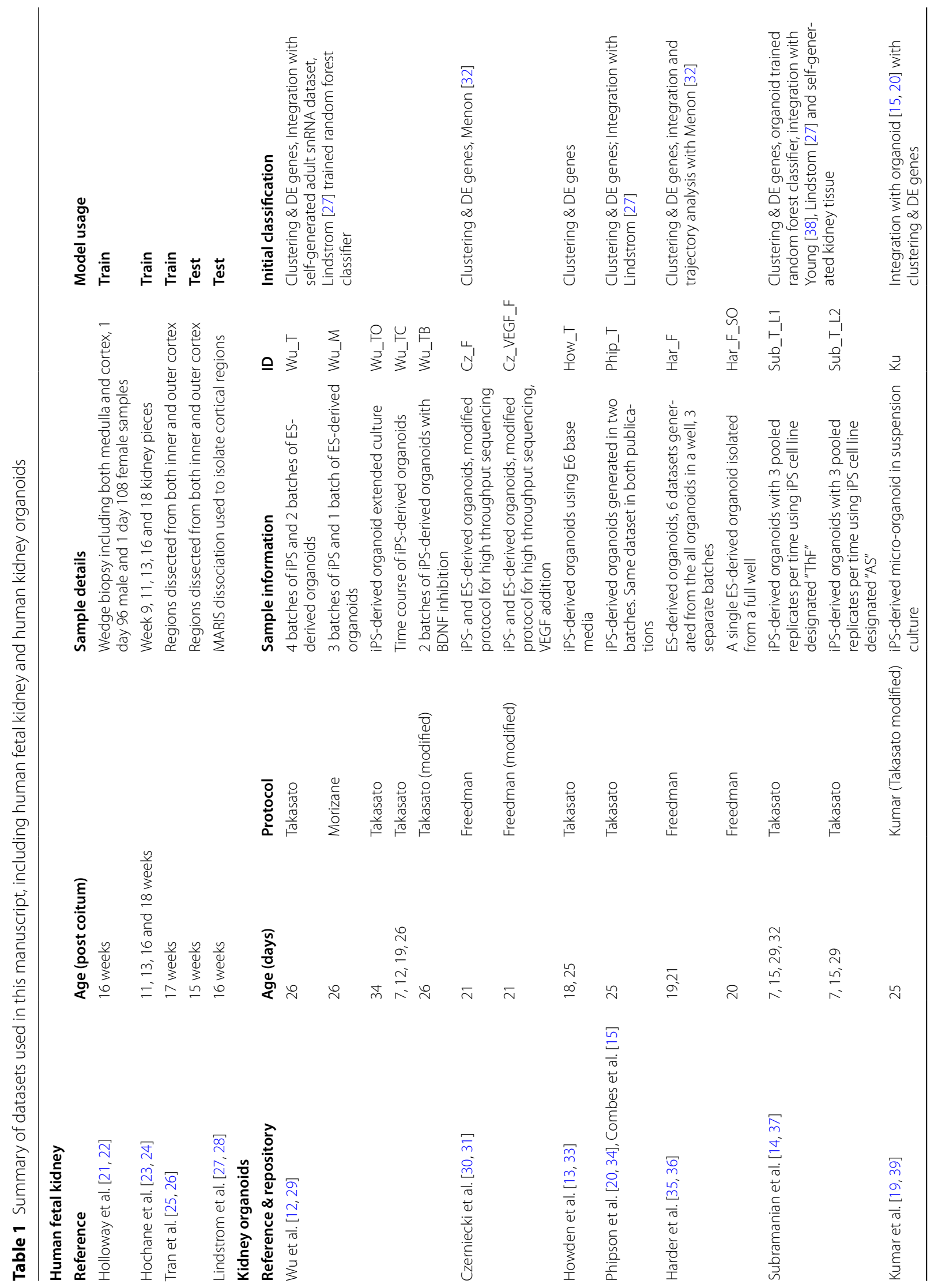




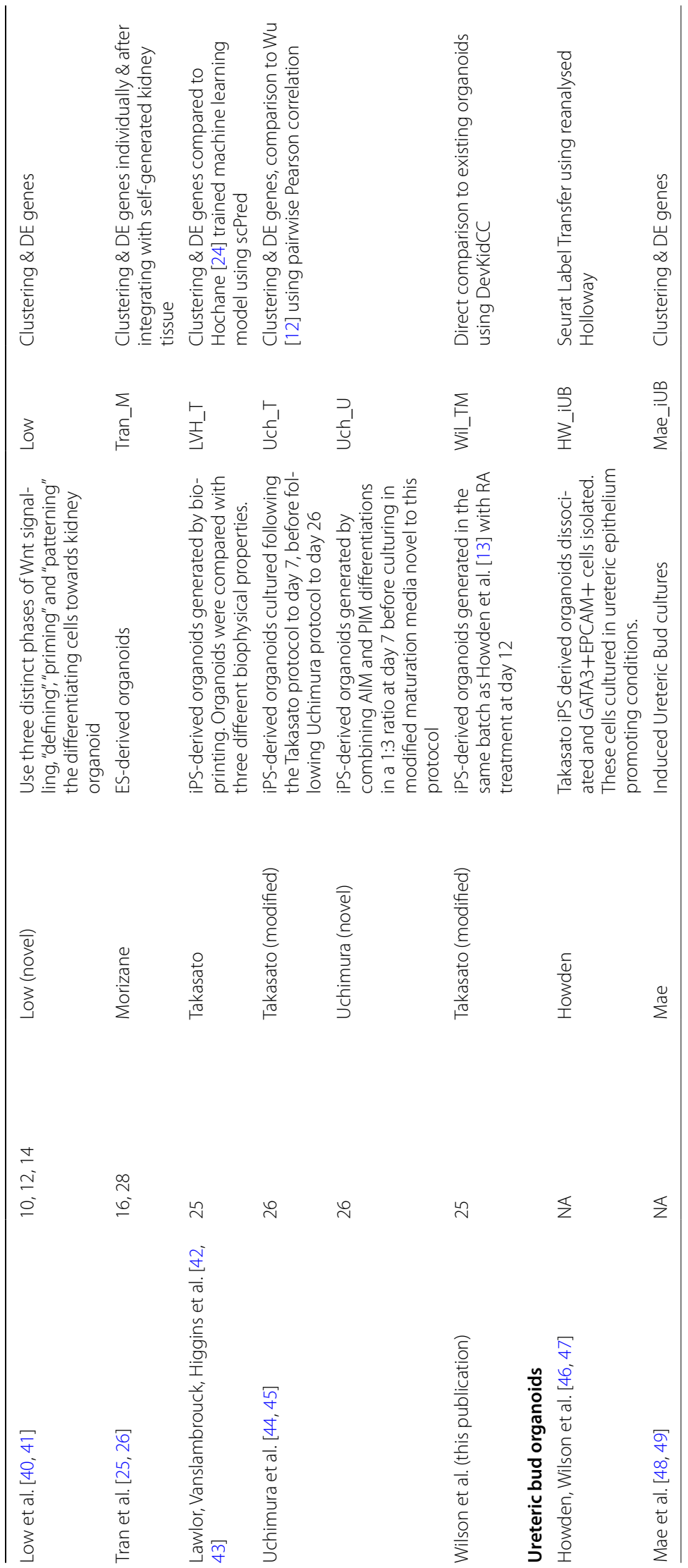


the cell types present within kidney organoids based on cell types within the developing human kidney.

Here we have taken reference HFK datasets from three publications that span multiple ages and kidney regions (Table 1), performed individual annotations of the cells present based on prior information, then used all confidently classified cells to train classification models using the $R$ package scPred [53], a generalisable method which has showed high accuracy in different experiments and datasets from multiple tissues, and considered a top performer in benchmarking studies [9]. We finally utilise established knowledge of kidney developmental biology to refine the classification of off-target cell types. The resulting model, referred to as DevKidCC, provides a robust and accurate classification of cells in novel singlecell datasets generated from developing human kidney or stem cell-derived kidney organoids. DevKidCC defines a model of cellular identity organised in a hierarchical manner to represent the key developmental trajectories of lineages within the developing kidney. The classification method is complemented with custom visualization tools in the DevKidCC package. This classifier was then used to investigate published kidney organoid datasets to compare organoid patterning and gene expression profiles across these datasets. We present a variety of applications of DevKidCC to the reanalysis of existing data. This analysis revealed differences in cell type proportions, nephron patterning and maturation between kidney organoid protocols. We also applied DevKidCC to investigate approaches for directed differentiation to one cell population, the ureteric epithelium, and dissect the effect of all-trans retinoic acid on nephron patterning and podocyte maturation. While DevKidCC is specifically trained on HFK for application to kidney organoid models, the development framework presented here could be applied for any tissue system to generate a cell classification model.

\section{Methods}

\section{DevKidCC algorithm}

DevKidCC (Developing Kidney Cell Classifier) is a function written in $R$ designed to provide an accurate, robust and reproducible method to classify single cell RNAsequencing datasets containing human developing kidney-like cells. The algorithm has two steps: data preprocessing and cell classification. Below we describe the development and utilisation of these steps.

\section{Data pre-processing}

The required input is a scRNA-seq dataset as a Seurat $[7,8]$ object. The first step is extraction of the raw count matrix, which is then normalised by dividing the total expression of each gene by the total gene expression per cell then multiplied by a scale factor of 10,000 and natural log-transformed with pseudocount of 1 .

\section{Cell classification}

We generated a comprehensive developing kidney reference single-cell dataset by harmonising the raw data from multiple high-quality human fetal kidney datasets. The annotation of the reference included three tiers with increasing specificity, with a clear hierarchical structure between the tiers. This dataset was then used to train machine learning models using the $R$ package scPred [53]. One model was trained for each node of identities within the classification hierarchy.

Utilising scPred [53] the classifiers were trained using the same parameters, with the relevant cells inputted for each. The feature space used was the top 100 principal components. The classifiers were trained using a support vector machine with a radial kernel using one round of harmonisation. The classifiers are stored as a scPred [53] object and can be used to classify cells within a Seurat $[7,8]$ object using the scPred [53] package. These classifiers will calculate the probability of a cell belonging to the trained identities within that classifier, giving a probability score between 0 and 1 for each identity. It will then assign an identity of the highest score above the set threshold or call the cell unassigned if no identity scores above the threshold. Classification is organised in a biologically relevant hierarchy so as to optimally and accurately identify the cellular identity of all analysed cells. All cells are first classified using the first-tier model, containing generalised lineage identities of stroma, nephron progenitors, nephron, ureteric epithelium and endothelium. After probability calculation using the first-tier model, cells that do not pass the threshold are classified as unassigned. The area under the AUROC and AUPRC to decide a threshold were determined using the MLeval $\mathrm{R}$ package from CRAN https://cran.r-project.org/web/ packages/MLeval/index.html [54]. The threshold is set to 0.7 by default but can be adjusted by the user, which can be useful if the user wants to classify cells with decreasing degrees of probability. NPC cells were subjected to further investigation by subsetting and reclustering at a resolution level of 0.5 using FindClusters and identifying the percentage of cells expressing $P A X 2$ with clusters below $30 \%$ being relabelled as NPC-like. Cells assigned to stroma, nephron and ureteric epithelium are passed into a second tier of classification specific to these identities. It is important to note that at the second and third classification tiers, there is no thresholding, i.e., all cells are assigned an identity with no cells classed as unassigned. The second-tier ureteric epithelium model is trained on the tip, cortical, outer and inner medullary cell identities. The second-tier stroma model is trained on the stromal 
progenitors, cortex, medullary and mesangial cell identities. The second-tier nephron model is trained on the early nephron, distal nephron, proximal nephron, renal corpuscle and nephron cell cycle population. The distal nephron, proximal nephron and renal corpuscle are then further classified into more specific identities in a third tier of models. The third-tier distal nephron model is trained on early distal/medial cells, distal tubule and loop of Henle cells. The third-tier proximal nephron model is trained on early proximal tubule and proximal tubule cells. The third-tier renal corpuscle model is trained on parietal epithelial cells, early podocytes and podocytes. Each stage of the classification step is recorded as a metadata column, as is the final classification for each cell. All the probability scores and tier classifications are readily accessible within the Seurat $[7,8]$ object for further analysis.

\section{Comprehensive reference generation}

Raw data was downloaded from GEO database from repositories GSE114530 [23] and GSE124472 [25] or provided to us directly by the authors, since made available at EMBL-EBI ArrayExpress under accession number E-MTAB-9083 [21]. The data as CellRanger output was read into $R$ and processed using Seurat [7, 8] (v3.2.2), using SCTransform [55] for pre-processing. Clustering and manual annotation were performed on each dataset individually, referring back to the original papers and using established markers enriched in clusters to classify each cluster. Once annotated, datasets were integrated using Harmony [56] with 100 PCAs and 10000 variable features.

\section{Organoid gene expression database}

All available single-cell RNA-sequencing kidney organoid datasets were downloaded (from Gene Expression Omnibus (https://www.ncbi.nlm.nih.gov/geo/) with accession numbers GSE118184, GSE109718, GSE119561, GSE114802, GSE115986, GSE132026, GSE124472, GSE152014, GSE161255, GSE152685, GSE131086) [25, $30,33-35,40,42,44,46,48])$ and used to build a database. This database was generated by running DevKidCC, extracting summaries of the gene expression information at each classification tier and combining these into a formatted table. This database can be used to directly compare gene expression between existing datasets, also novel datasets classified using DevKidCC. The link to download this database is available at https://kidneyrege neration.github.io/DevKidCC/index.html [57].

\section{DevKidCC Kidney Organoid Gene Explorer shiny app}

To make visualisation of the organoid database possible outside of using $R$, a shiny app was developed that provides an interface to interact with the organoid database using a modified CompareDotPlot function. This allows for an interactive way to visualise and analyse gene expression within published organoid datasets. This app is accessible at https://kidneyregeneration.github.io/ DevKidCC/articles/ShinyApp.html [58].

\section{Downstream visualisation functions}

To facilitate data visualisation and analysis of DevKidCC classified datasets, three customised functions were included in the package. DotPlotCompare is a modified version of the DotPlot function from the Seurat package. A gene expression profile of the reference is present within the function and can be used for direct comparisons to an existing or novel dataset. There is an option to visualise the organoid database within this function as well; the downloading instructions for this are available at the package Github repository (https://github.com/Kidne yRegeneration/DevKidCC) [57]. The proportions of cells classified using DevKidCC can be visualised as a bar chart using the ComparePlot function. This can also take as input a gene and show the expression of that gene in each segment. The IdentMeans function produces summarises the contribution of samples to each population through a chart showing the mean and standard deviation/standard error of the mean.

\section{iPSC-derived organoid differentiation}

The day prior to differentiation, CRL2429-MAFBmTagBFP2/GATA3mCherry human iPSCs [59] or CRL2429SIX2EGFP [13, 59] were dissociated with TrypLE (Thermo Fisher Scientific, cat\# 12563029), counted using a haemocytometer and seeded onto Laminin 521-coated (Biolamina, cat\# LN-521-03) 6-well plates at a density of $50 \times 10^{3}$ cells per well in Essential 8 (Thermo Fisher Scientific, cat\# A1517001) medium. Intermediate mesoderm induction was performed by culturing iPSCs in TeSR-E6 medium (Stem Cell Technologies, cat\# 05946) containing 4-8 $\mu \mathrm{M}$ CHIR99021 (R\&D Systems, cat\# 4423) for 4 days. On day 4, cells were switched to TeSR-E6 medium supplemented with $200 \mathrm{ng} / \mathrm{ml}$ FGF9 (R\&D Systems, cat\# 273-F9-025) and $1 \mu \mathrm{g} / \mathrm{ml} \mathrm{Heparin} \mathrm{(Sigma-Aldrich).}$ On day 7, cells were dissociated with TrypLE, diluted five-fold with TeSR-E6 medium, transferred to a $15-\mathrm{ml}$ conical tube and centrifuged for $5 \mathrm{~min}$ at $300 \mathrm{x} g$ to pellet cells. The supernatant was discarded, and cells were resuspended in residual medium and transferred directly into a syringe for bioprinting. Syringes containing the cell paste were loaded onto a NovoGen MMX Bioprinter, primed to ensure cell material was flowing, with 100,000 cells deposited per organoid onto a 0.4- $\mu \mathrm{m}$ Transwell polyester membranes in 6-well plates (Corning). Following bioprinting, organoids were cultured for $1 \mathrm{~h}$ in 
presence of $6 \mu \mathrm{M}$ CHIR99021 in TeSR-E6 medium in the basolateral compartment and subsequently cultured until day 12 in TeSR-E6 medium supplemented with $200 \mathrm{ng} /$ $\mathrm{ml} \mathrm{FGF9}$ and $1 \mu \mathrm{g} / \mathrm{ml} \mathrm{Heparin.} \mathrm{From} \mathrm{day} 12$ to day 25, organoids were grown in TeSR-E6 medium either without additional supplement or with additional $5 \mathrm{uM}$ alltrans retinoic acid (Sigma-Aldrich, cat\# R2625-100MG). Unless otherwise stated, kidney organoids were cultured until harvest at day 25 .

\section{Flow cytometry}

Prior to analysis, single-kidney organoids were dissociated with $0.2 \mathrm{ml}$ of a 1:1 TrypLE/Accutase solution in 1.5$\mathrm{ml}$ tubes at $37^{\circ} \mathrm{C}$ for $15-25 \mathrm{~min}$, with occasional mixing (flicking) until large clumps were no longer clearly visible. $1 \mathrm{ml}$ of HBBS supplemented with 2\% FBS was added to the cells before passing through a 40-1M FACS tube cell strainer (Falcon). Flow cytometry was performed using a LSRFortessa Cell Analyzer (BD Biosciences). Data acquisition and analysis were performed using FACSDiva (BD) and FlowLogic software (Inivai). Gating was performed on live cells based on forward and side-scatter analysis.

\section{Whole mount immunostaining}

Fixed kidney organoids were incubated in blocking buffer (PBS 1X donkey serum $10 \%$ triton $\mathrm{X} 1000.3 \%$ ) at $4^{\circ} \mathrm{C}$ for $3 \mathrm{~h}$ before adding primary antibodies against HNF4 $\alpha$ (Life Technologies 1:300, cat\# MA1-199), Nephrin (NPHS1 1:300, Bioscientific, cat\# AF4269) and Claudin-1 (CLDN1 1:100, Thermo Fisher Scientific, cat $\# 71-7800$ ) at $4^{\circ} \mathrm{C}$ for 2 days. After washing in PBS $1 \mathrm{X}$ triton X-100 0.1\%, organoids were incubated in secondary antibodies 1:400 at $4^{\circ} \mathrm{C}$ for 2 days: Alexa fluor 405 donkey anti-mouse (Abcam, cat\# ab175659), Alexa fluor 488 donkey antigoat (Molecular Probes, cat\# A11055) and Alexa fluor 568 donkey anti-rabbit (Life Technologies, cat\# A10042). Samples were then washed before blocking at $4^{\circ} \mathrm{C}$ for $3 \mathrm{~h}$ with PBS $1 \mathrm{X}$ mouse serum $10 \mu \mathrm{g} / \mathrm{ml}$ triton X-100 0.3\% and adding an APC-conjugated CD31 antibody (1:50, Biolegend, cat\# 303115 ) at $4^{\circ} \mathrm{C}$ for 2 days. Finally, samples were washed and imaged in 50:50 glycerol:PBS $1 \mathrm{X}$ using a Dragonfly Spinning Disc Confocal Microscope (Andor Technology).

\section{Single-cell transcriptional profiling and data analysis}

The novel dataset presented in this paper was generated from the same batch of samples presented in Howden et al. [13]. Human iPSC organoids were dissociated as described above (for flow cytometry) and passed through a 40- $\mu \mathrm{M}$ FACS tube cell strainer. Following centrifugation at $300 \mathrm{~g}$ for $3 \mathrm{~min}$, the supernatant was discarded and cells resuspended in $50 \mu \mathrm{l} \mathrm{TeSR-E6} \mathrm{medium.} \mathrm{Viability} \mathrm{and}$ cell number were assessed, and samples were run across separate runs on a Chromium Chip Kit ( $10 \times$ Genomics). Libraries were prepared using Chromium Single-Cell Li sequenced on an Illumina HiSeq with 100-bp paired-end reads. Cell Ranger (v1.3.1) was used to process and aggregate raw data from each of the samples returning a count matrix. Quality control and analysis was performed in $R$ using the Seurat package (v3.2.2). 1668 cells expressing more than 1500 genes and less than 30\% mitochondrial genes passed quality control with means of 15100 for UMI count and 3697 for genes expressed. Classification was performed using DevKidCC (v0.1.6) as described in this manuscript.

\section{Results \\ Generation of the model hierarchy for complete cell classification}

We first built a comprehensive reference dataset on which to train the probabilistic classification models. We used high quality HFK single-cell RNA-sequence datasets published in Hochane et al. [24], Tran et al. [26] and Holloway et al. [22] (Table 1). Samples ranged from 9 to 19 weeks' gestation, across which time the developing human kidney undergoes both growth and maturation, with week 16 being most frequently represented. These references were originally annotated using clustering and cluster labelling using marker gene expression. One dataset was a recently published high quality HFK dataset [22] (8,987 cells) that included both medulla and cortex regions and including a 96-day male and 108-day female sample. Of note, this dataset contained ureteric epithelium, which had not been thoroughly analysed to this point [47]. This data was combined with data from 17,759 HFK cells ranging from week 11 to 18 of gestation [24] to increase the developmental range of the training set. A further 8317 cells from gestational week 17 which had been microdissected into the cortex, inner and outer medullary zones [26] were combined to complete the comprehensive reference single-cell RNA-sequencing HFK dataset. Cells from all datasets were integrated using Harmony [56] (Fig. 1A) before performing a supervised clustering and annotation, using the original annotations of each dataset as a guide. This led to a reference dataset containing three ureteric epithelial subpopulations including ureteric tip (UTip), outer stalk (UOS), inner stalk (UIS), four stromal subpopulations including stromal progenitor cells (SPC), cortical stroma (CS), medullary stroma (MS), mesangial cells (MesS), endothelium (Endo), the nephron progenitor cells (NPC) and the nephron including subpopulations of early nephron (EN), early distal tubule (EDT), distal tubule (DT), Loop of Henle (LOH), early proximal tubule (EPT), proximal tubule (PT), parietal epithelial cells (PEC), 


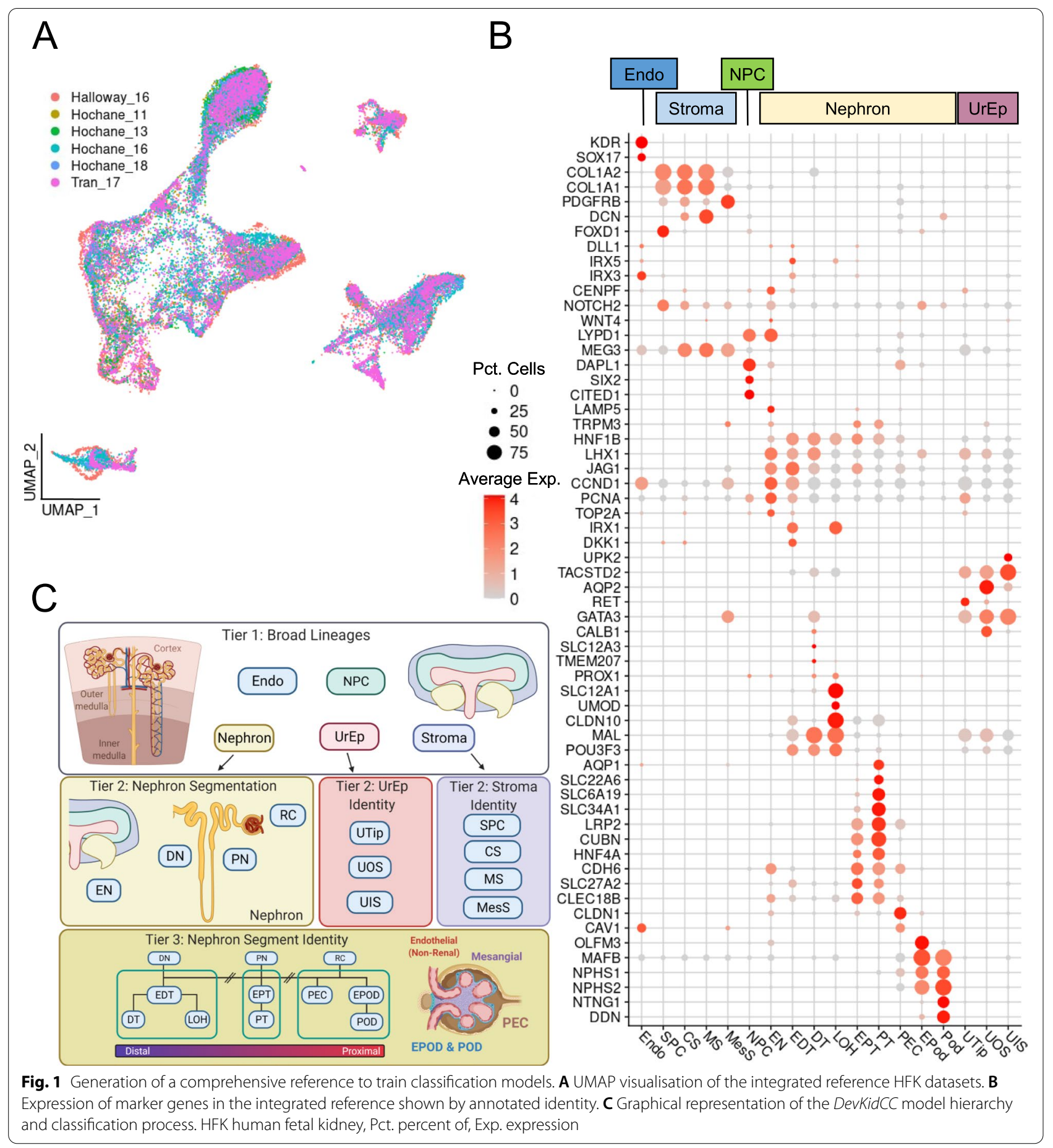

early podocytes (EPod) and podocytes (Pod) (Fig. 1B, C, Additional file 1: Fig. S1). Some of these populations were further classified in the original publications, including the DT being split into distal straight, distal convoluted and connecting segment or the classification of populations in relation to morphological features, such renal vesicle, comma shaped body and S-shaped body segmentation [24, 26, 47]. While morphologically there is a consistency in segment identification, this is less clear in single-cell data and has led to inconsistency in classification terminology. As such, here we have classified cell populations based on 
expression of known differentiation markers as cells take on a more distinct identity.

\section{scPred-derived models provide accurate classification of kidney cell types}

The complex and dynamic nature of the developing kidney, with multiple cell lineages and waves of nephrogenesis, means that cells of many stages of differentiation can be present at all developing timepoints within the same single-cell data. This is one of the main challenges in classifying cells in the HFK single-cell data, as the cells are in transitional flux. The multiple lineages within the kidney also make classifying cell types difficult, as the differences between lineages mask the subtle differences in gene expression between cell types within a lineage, such as those of the epithelial sub-types. To minimise the impact of this transcriptional variance on classification, we took a hierarchal approach by using annotations at differing degrees of resolution to train three tiers of models (Fig. 1C). The models were trained with the package scPred [53] which utilises a machine learning approach to train sets of binary predictive models on a reference single-cell dataset. This model estimates the probability of a cell within a query dataset belonging to an identity group classified within the model. This has been shown to be a robust method to classify cells of a novel dataset based on a known reference $[9,43,53]$. The advantages of using scPred include ready integration with Seurat objects and the capacity to utilise many machine learning models available through the caret package. scPred provides ROC, sensitivity and specificity metrics using held-out training data for each binary classifier within a model which we used to benchmark multiple classes of models. A support vector machine with a radial basis kernel (svmRadial) and 100 principal components was used, with this performing equal to or better than a generalised linear model (glm) or neural network implementation (nnet) (Table S1A).

When implementing probabilistic models in practice, it can be beneficial to establish a threshold for a cell to be assigned an identity; however, this is difficult as organoids do not have a 'ground truth' for cell identity. While scPred provides ROC, sensitivity and specificity metrics using held-out training data for each binary classifier within a model (Table S1B), we wanted to investigate the model's accuracy on organoid datasets. For this, we used two organoid single-cell datasets to test the binary classifiers within the tier 1 model which classified cells based on their lineage; nephron progenitor cells (NPC), nephron, ureteric epithelium (UrEp), stroma and endothelial. The Howden et al. [13] organoids were used as we had access to the original annotation showing a representation of all key cell types, while the Uchimura et al. [45] dataset was reported to be enriched for the UrEp population. We used the standard clustering pipeline to reproduce Uchimura annotation from the original publication. While the AUROC was 0.93 or higher for all tests, the AUPRG curves showed a much faster drop off in precision for the Howden et al. [13] test than Uchimura et al. [45] (Additional file 1: Figure S2A). Using the performance of the Nephron, NPC, UrEp and Stroma binary classifiers in these tests led to setting a default threshold of 0.7 for the tier 1 model's classification with all cells having a maximum probability below this remaining 'unassigned.'

We next investigated the probability scores of the model on all freely available published organoid datasets (371,570 cells from 58 samples) ranging from 7 to 32 days of culture and two human fetal kidney datasets including the frequently used Lindstrom et al. [27]. While the distribution of the maximum scores for cells in the HFK and organoid datasets showed very similar patterns, organoids showed a lower mean and larger SEM (Fig. 2A). A two-sample $t$ test comparing the HFK and organoid probability scores of 'end-stage' organoids, i.e., those beyond 18 days of culture, indicated significant differences between the assigned nephron $\left(p<2.3 \times 10^{-36}\right)$, UrEp $(p$ $\left.<2.9 \times 10^{-25}\right)$, stroma $\left(p<2.3 \times 10^{-308}\right)$ and NPC $(p<2.3 \times$ $\left.10^{-308}\right)$. The model classified between $60.8 \%$ and $92.0 \%$ of 'end-stage' organoids, while the HFK samples had $>90 \%$ classification (Fig. 2A). The 'unassigned' cells may represent non-renal off target cell types not normally present in HFK or cells in which identity is not sufficiently strong for definitive classification. When applied to the dataset of Lindstrom et al. [27], this model classified $90.4 \%$ of the 2945 cells that passed quality control, while the remaining cells expressed markers for immune cells (HLA-DRA, CCL3, SRGN) which are not represented in the model and so were not assigned an identity (Additional file 1: Figure S2B,C). 14 cells (0.5\%) were classified as UrEp, positioned at the tips of one end of the nephron cluster. The nephron cells nearest to the UrEp population were further classified as DN epithelium (not shown). While these two cell populations arise from distinct precursors,

(See figure on next page.)

Fig. 2 DevKidCC accurately classifies human fetal kidney data. A Probability score distributions for the tier 1 classifier for both human fetal kidney (left) and organoid (right) data, grouped by tier 1 classification. B Mean number of cells expressing shown genes, grouped by HFK NPCs, organoid NPCs, organoid NPC-like and organoid unassigned populations. C Probability score distribution for the tier 2 stroma classifier on all organoids. D Probability score distribution for the tier 2 UrEp classifier on all organoids. E Probability score distribution for the tier 2 and 3 nephron lineage classifiers on all organoids 
A

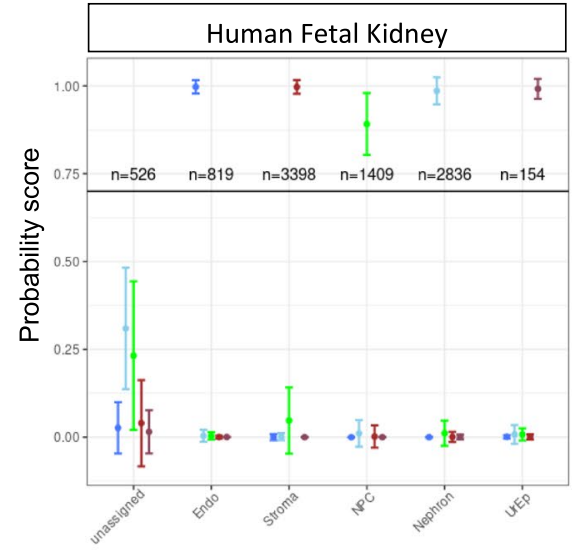

B

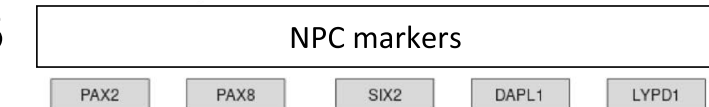

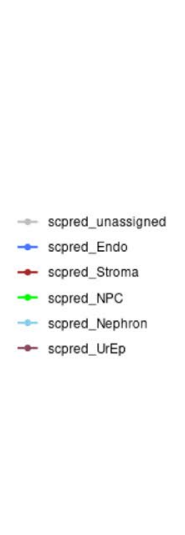
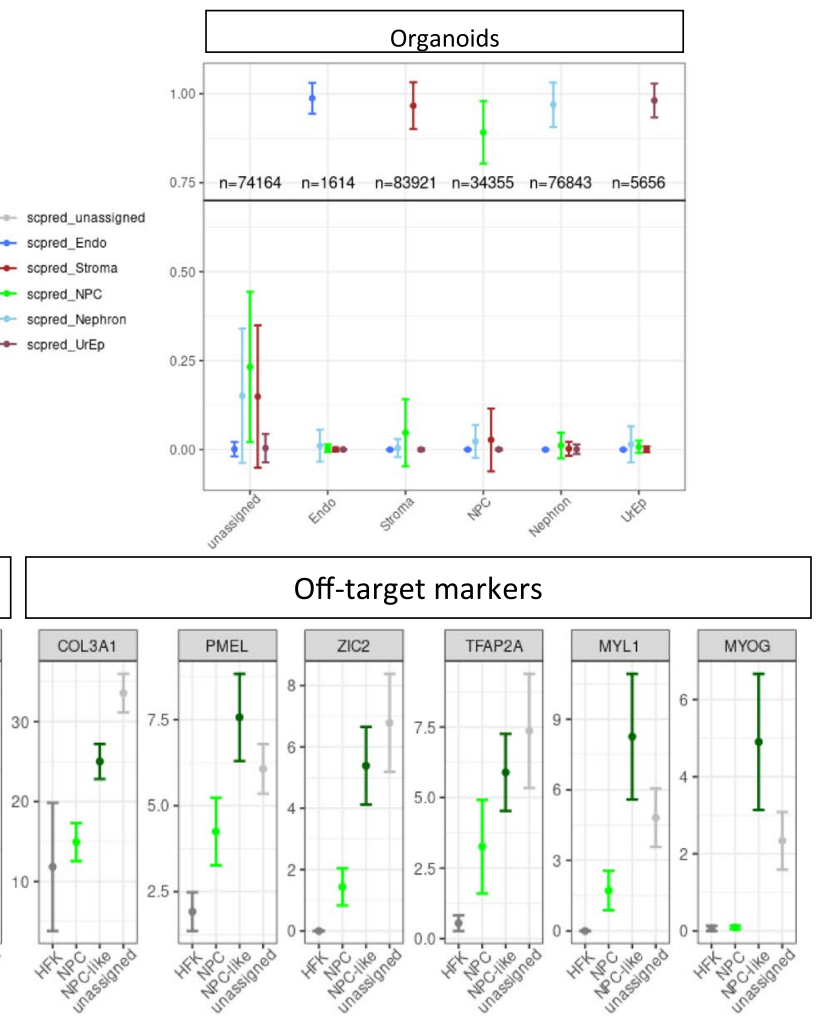

Identity $\rightarrow$ NPC $\rightarrow$ NPC-like $\rightarrow$ unassigned
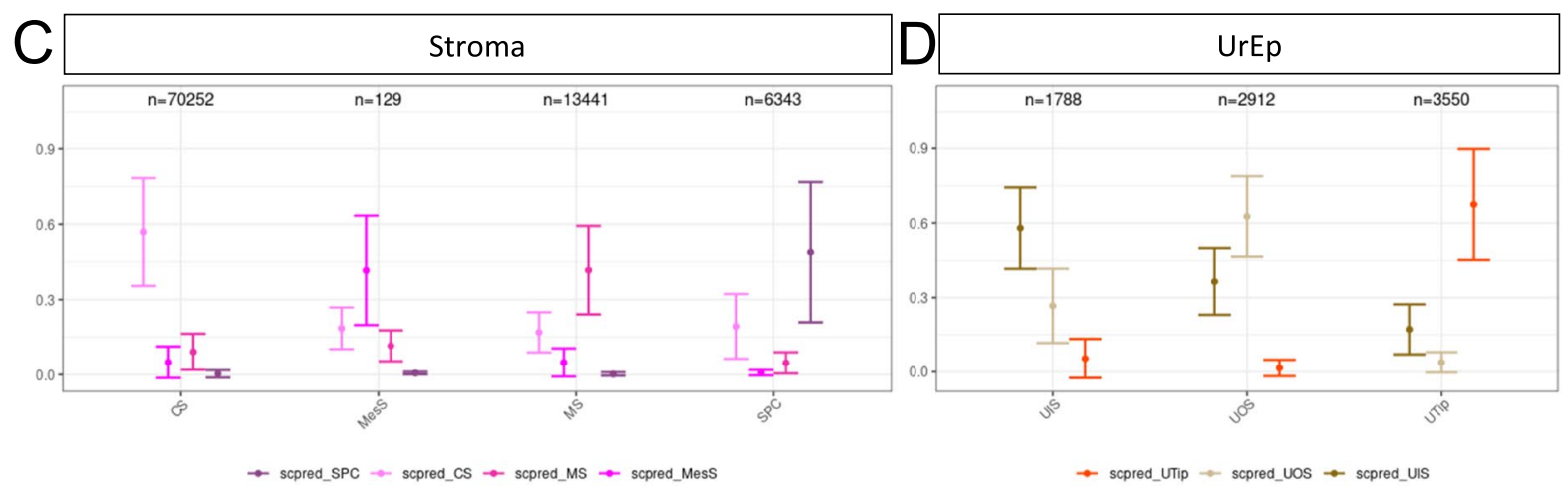

$\rightarrow$ scpred_UTip $\rightarrow$ scpred_UOS $\rightarrow$ scpred_UIS

E Nephron

Nephron: DN
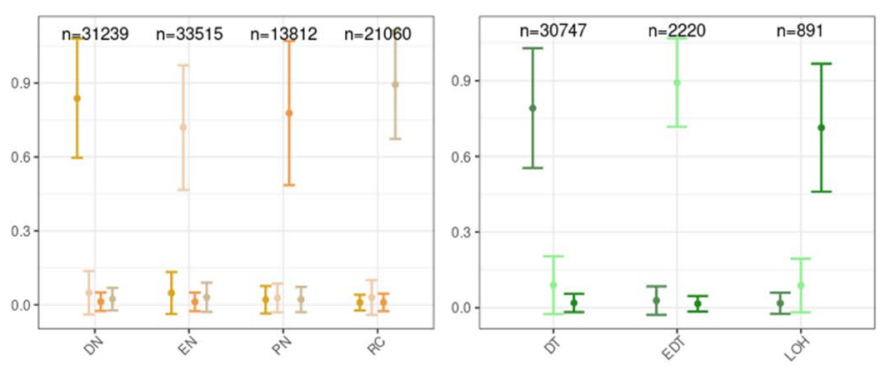

- scpred_EN $\rightarrow$ scpred_DN $\rightarrow$ scpred_PN $\rightarrow$ scpred.

$\rightarrow$ scpred_EDT $\rightarrow$ scpred_DT $\rightarrow$ scpred_LOH

Fig. 2 (See legend on previous page.) 
they share a similar transcriptional profile, making them difficult to distinguish at single-cell level [15-18, 47]. The ability to identify and classify these two populations separately, even with a small contribution of one population within a dataset, demonstrates the power of using scPred-derived models. The expression of marker genes used by Lindstrom et al. [27] to annotate cell identities were shown as enriched in the same populations classified using DevKidCC (Additional file 1: Figure S2C), affirming the accuracy and relevance this classification method.

Some samples showed classified NPC populations with enrichment for 'off-target' markers of muscle (MYL1, MYOG) neural (ZIC2) and melanin-expressing $(P M E L)$ populations, in line with previous reports (Additional file 1: Fig. 2D). This is particularly relevant as the Howden et al. [13] dataset used for model evaluation is one of these. One theory for the generation of these cell types is misdirected differentiation potentially from a shared progenitor with the NPC cells that arise, which may explain the similarity to the NPC profile and high probability score. $P A X 2$ has been shown as a gene that marks the 'lineage boundary' between the NPC and stromal populations in vivo [60], as well as being an early marker of the nephron lineage [61]. There is evidence, however, that $P A X 2$ is dispensable for in vitro nephron formation [62], once again highlighting differences between in vivo and in vitro systems. We investigated the expression of $P A X 2$ in the NPC classified cells and found a correlation between $P A X 2$ expression and other key markers of NPCs such as LYPD1 and SIX2. There is also an inverse correlation between $P A X 2$ expression and offtarget markers of muscle, neural and melanin-expressing cells (Fig. 2B). Incorporating this biological knowledge to refine the classification of NPCs, we included a step to subcluster and screen for $P A X 2$ expression, with subclusters having less than $30 \%$ of cells express $P A X 2$ being relabeled as 'NPC-like' (Fig. 2B). These cells may have the potential to undergo nephrogenesis if correctly induced however lack a clear in vivo NPC transcriptional signature, making them likely in vitro artefacts arising in this system.

The cells classified as nephron, stromal and UrEp underwent the further stage/s of classification. The stromal and UrEp population utilise one further classifier, classifying them into stromal subsets of SPC, CS, MS and MesC (Fig. 2C), while the UrEp population is further classified into UTip, UOS and UIS identities (Fig. 2D). The nephron population however has additional segmentation and requires two further stages of classification (Fig. 2E). A summary of the scPred provided AUROC, sensitivity and specificity metrics generated using heldout training data for each binary classifier within a model, which range between 0.879 and 1.000 , are provided in Additional file 3: Table S2. However, lack of a 'ground truth' for organoid identity makes it difficult to precisely evaluate model performance using held out in vivo data. To complement that analysis, we compare the probability scores for all tests within an identity (Fig. 2C-E). These results highlight the accuracy of these additional models, particularly within the nephron cell identities.

These models are utilised in a hierarchical method of classification provided in a single-call wrapper function $D K C C($ ) within the R package DevKidCC, taking an input a Seurat object. To determine cells in the first tier, a probability threshold of 0.7 is set while at all other tiers the threshold is removed. This enables all cells that are classified at the top tier to be given an assigned identity regardless of the highest degree probability predicted by the lower tier models. Further investigation of the calculated probability can be interrogated as every cell has a record in the metadata of the scores from each classification. No preprocessing is required as $D K C C()$ utilises Seurat's NormalizeData() function for data normalisation. The recommended pipeline is to read in raw counts data using the Seurat pipeline, filter out poor quality cells and then run $D K C C()$. The classifications for each tier and the final identities can be accessed within the metadata slot for further investigation. The package contains custom in-build functions ComparePlot, DotPlotCompare and IdentMeans to investigate the cell populations within the classified sample.

\section{DevKidCC classification rapidly and accurately reproduces published annotations}

To investigate the utility of using this package on realworld data, the DevKidCC classification of two published kidney organoid single-cell datasets was compared to their original cluster-based annotations. Howden et al. [13] contained samples from two differentiation timepoints; intermediate (18 day) and late (25 day) stage organoids while $\mathrm{Wu}$ et al. [12] contained day 26 organoid datasets from two distinct protocols for deriving kidney organoids, labelled as Takasato [63] and Morizane [64] after the original authors. Within the Howden et al. [13] and Wu et al. [12] data, $76.0 \%$ and $61.1 \%$ of cells were assigned using DevKidCC, respectively. Within the Howden et al. [13] dataset, all original clusters contained cells that were reclassified as unassigned, with the largest contribution being from clusters previously annotated as the neuron and muscle, illustrating the specificity with which the model classifies renal cell types (Fig. 3A).

Both stroma and NPC are mesenchymal cell types. The mesenchymal cells present within kidney organoids have been difficult to accurately classify due to their gene expression profiles being different to those of 


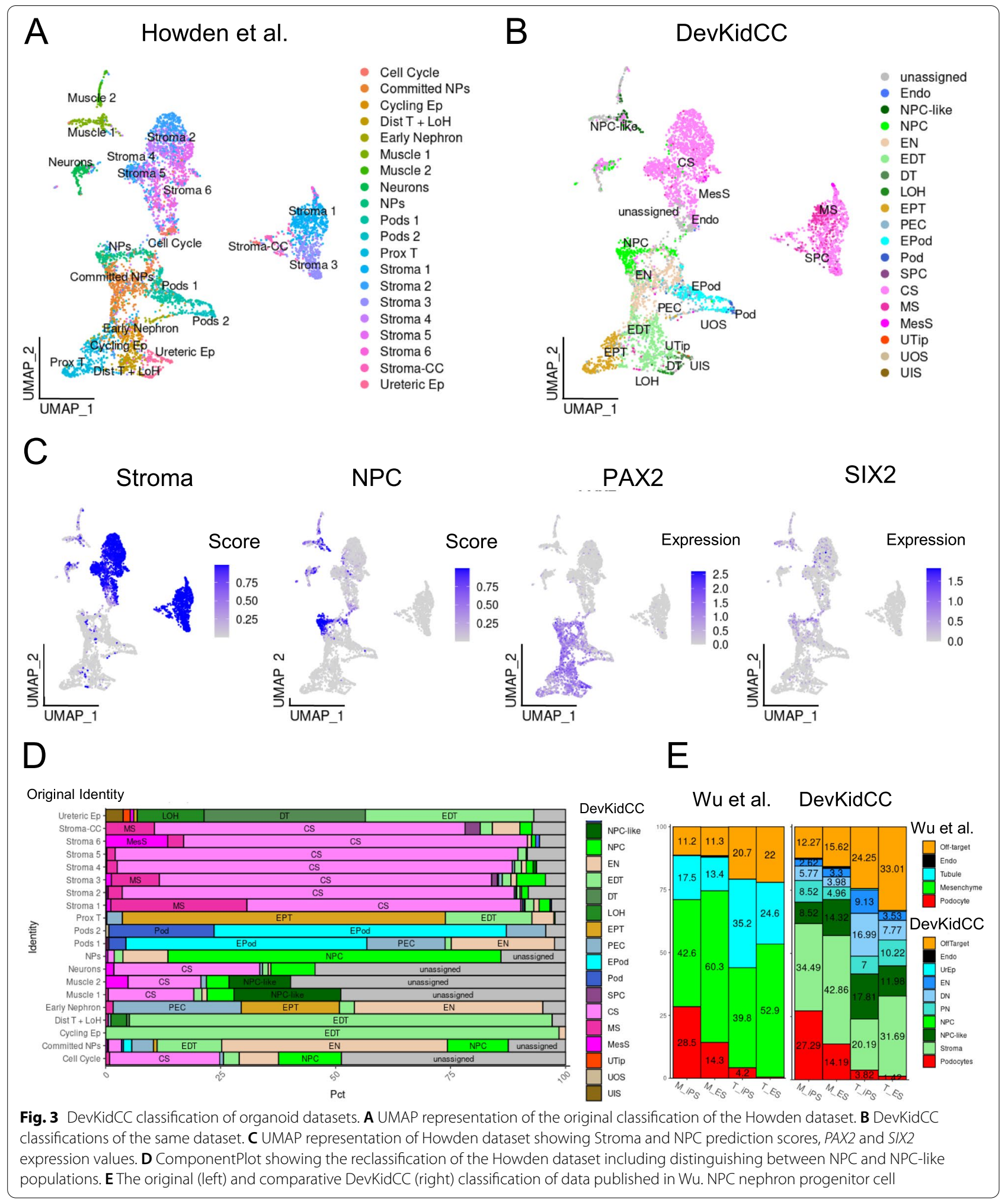

characterised developing kidney stroma [15]. There was some overlap in the distribution of cells with high probability for both stroma and NPC (Fig. 3C). Cells within organoids that share expression profiles both with stroma and NPC have been previously noted [16] and may arise as an in vitro artefact. The $P A X 2$ lineage differentiation 
between NPC and NPC-like cells is clearly shown when comparing the high probability NPC cells expressing $P A X 2$ being localised to the larger 'nephron' cluster while the high probability NPCs not expressing $P A X 2$ are separately localised in distinct clusters (Fig. 3C). The expression of SIX2, the gold standard in vivo NPC marker, is shown for comparison and has a wider distribution including other mesenchymal cell types labelled as Stroma, in line with the initial publication of this data (Fig. 3C). DevKidCC reclassified $\sim 25 \%$ of cells originally annotated as 'muscle' and 'neural' as NPC or NPC-like, while also reclassifying some as stromal cells. The offtarget populations were noted to share expression of key NPC markers such as SIX2 and SALL1 [13] indicating there is some transcriptional similarity. Within the nephron, cells previously identified as 'Committed and Early Nephron' due to the expression of both committed NPC (LYPD1) and early nephron (LHX1, JAG1) markers within this cluster were reclassified by DevKidCC to distinguish between the two cell populations (Fig. 3D). The previous analysis of the Howden et al. [13] data identified seven clusters as stromal (Fig. 3A, D), of which almost all of those assigned an identity using DevKidCC remained classified as a stromal sub-type (Fig. 3D).

To further examine the capability of DevKidCC classification, we analysed organoid datasets from $\mathrm{Wu}$ et al. [12] generated from either embryonic (ES) or induced pluripotent (iPS) stem cells using two different protocols [12]. Using DevKidCC with default parameters, we were able to rapidly reproduce the initial classification of these organoids, accounting for the differences in the nomenclature (Fig. 3E). This classification identified an increased population of cells not matching the reference (termed 'unassigned') compared to the original annotation. Here, DevKidCC could again distinguish kidney cells from likely off target cell types, such as the originally reported neural population, that may represent artefacts of in vitro culture $[12,13]$. This demonstrates how DevKidCC provides a consistent and measurable benchmark for kidney cell classification in organoids that can be applied to all data, enabling direct and relevant comparisons. Together these reanalyses demonstrate the accuracy with which DevKidCC can classify renal cell types within organoid datasets.

\section{DevKidCC provides a method for direct comparison between protocols}

A major challenge for the field has been to compare between datasets generated from different labs, lines, batches or from different protocols due to differences in the analyses that were used. This is particularly pertinent given the use of several distinct protocols for generating kidney tissue from hPSCs (see Table 1). Direct comparisons between studies and protocols requires an integration of all existing samples to allow re-clustering and differential gene expression analysis on the combined dataset. This is challenging due to the noise between samples, the majority of which relates to technical or batch effects [20] that can confound biological variations of interest during data integration [65]. To avoid these challenges, DevKidCC was used to directly identify all cell types present within multiple datasets enabling direct comparisons without the need for integration. As DevKidCC will compare all cells to the same comprehensive reference, the biological information for each sample can be directly compared without prior dimensional reduction and clustering. To demonstrate this, we applied DevKidCC to all available single cell kidney organoid datasets (summarised in Table 1) irrespective of the cell line, organoid age, differentiation protocol or laboratory. This comprehensive analysis allows a direct comparison of cell proportions across all samples at each tier of classification. We first focused on end stage organoids from the three main differentiation protocols represented in the literature, Takasato et al. [63], Morizane et al. [64] and Freedman et al. [66] (Fig. 4A). This immediately showed variation in the proportions of 'unassigned' cells across all datasets and the lack of nephron maturation even in the oldest organoids regardless of protocol. The maturation of nephron cell types was limited in all protocols and samples, although the Morizane [64] protocol produced organoids with the highest number of cells reflective of a more mature podocyte stage. While there were a small number of mature podocytes, there were almost no mature proximal tubule cells generated with any organoid protocol, with cells rather being classified as less mature EPT with expression of proximal markers such as CUBN, LRP2 and HNF4A but lack the specific solute channels such as SLC47A1, SLC22A2 and $S L C 22 A 8$ (Additional file 1: Figure S3). In clusteringbased analyses, these cell populations are often split into two or more groups which are interpreted to have varying degrees of maturation, whereas the DevKidCC classification indicates that these are mostly immature. There is noticeable variance between publications generating organoids from the same protocol, concurring with earlier studies showing that batch differences are a notable source of variation $[12,20]$. We performed two analyses of the unassigned populations, grouped by protocol. The most conserved differentially expressed genes between samples of each protocol were inputted into the ToppFun browser, with the Takasato protocol [63] and Morizane protocol [64] derived populations showing similar upregulated pathways such as skeletal system development and extracellular matrix organization, while the Freedman protocol [66]-derived populations showed an enrichment 


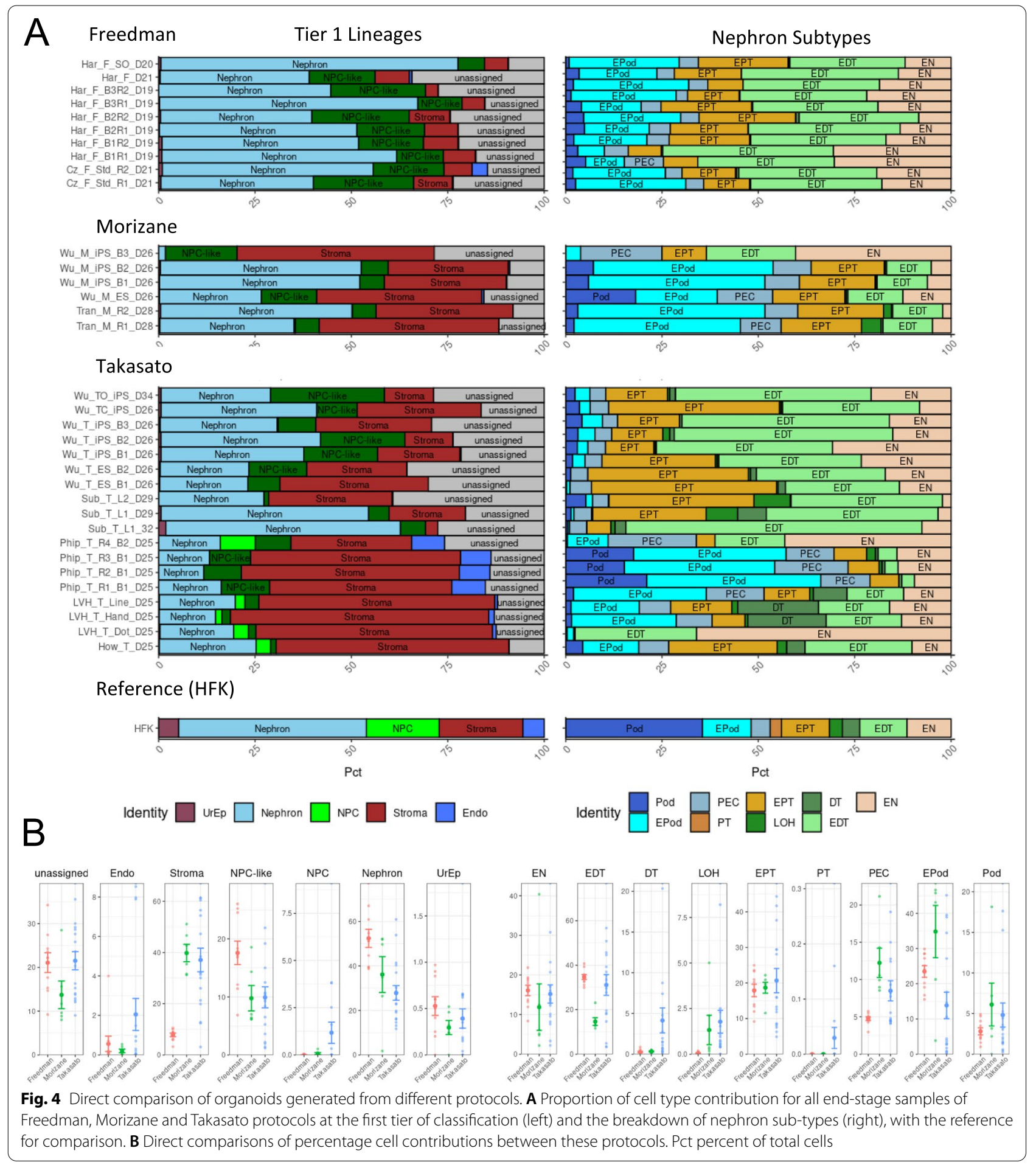

for neural system pathways (Additional file 1: Figure S4A). A second independent analysis used the Azimuth web browser to annotate these cells using the human fetal development reference. This analysis predicted the identity of each cell, with the strongest probabilities falling in the skeletal muscle and satellite cell categories in all protocols, with a range of other cell types being predicted, including populations of metanephric and ureteric cells of the kidney, various stromal populations and some neural subtypes (Additional file 1: Figure S4B). Interestingly, 
$37.47 \%$ and $23.38 \%$ of these cells derived from Morizane [64] and Freedman [66] protocols, respectively, were assigned metanephric while only $0.89 \%$ of Takasato [63]-derived cells. This analysis highlights the variation in the transcriptomic profiles present within organoid populations and the challenges in identifying those cell types even with the best available analysis pipelines.

DevKidCC analysis revealed differences in cell proportion and nephron patterning between organoids generated with different protocols. The proportion of cell populations within the reference HFK is unlikely to be representative of the ratios within a developing kidney due to the different methods used to collect samples; however, as organoid samples are typically a whole sample dissociation, we can infer some information comparing between these. Organoids generated using the Freedman protocol [66] show a small stromal population in comparison to other protocols while containing more early-stage nephron cells, although this may be indicative of the slightly younger age of these organoids. In the Morizane protocol [64] organoids, we identify limited distal tubule cells, with less than $25 \%$ of the nephrons classified as distal whereas the Takasato [63] and Freedman [66] protocols show more evenly segmented nephron components. The Takasato protocol [63] generates the most distal tubule (Fig. 4B), including some cells classed as a more mature DT segment as well as an $S L C 12 A 1$ expressing Loop of Henle population. The DT expressed GATA 3 and TMEM $52 B$ but lacked the distal convoluted tubule specific marker $S L C 12 A 3$, although in some cases the connecting segment specific marker CALB1 is expressed (Additional file 1: Figure S4). This would indicate that the connecting segment, which represents the most distal region of the nephron and which invades and fuses into the ureteric tip to form a contiguous tube, is being generated in some organoids. This is promising as it would indicate that there is the potential to promote fusion of these nephrons to any separately induced collecting duct structure, potentially enabling kidney tissue engineering. In summary, while nephrons are forming and showing evidence of patterning and identifiable segmentation in all protocols, their relative proximo-distal patterning and evident immaturity will impact their utility for disease modelling and drug screening studies.

\section{Identifying nephron progenitor cell variation between protocols}

To further investigate relative gene expression between datasets, we extracted gene expression profiles and proportions of cells in each classified population, in all available organoid datasets (see Table 1) and the comprehensive reference. A modified version of the DotPlot function from the Seurat $[7,8]$ package was included to directly compare gene expression between datasets and the reference. The NPC are a crucial population when considering kidney organoids as in vivo they give rise to the entirety of the nephrons [67], the functional unit of the adult kidney. Our classification system has highlighted the difference between NPC and NPC-like cells that arise during in vitro differentiation; however, we sought to further investigate the NPC population within organoids. The direct comparison between kidney organoids (Fig. 4A, B) revealed substantial variation in the proportion of NPCs, which we further investigated by applying the function DotPlotCompare (modified DotPlot from the Seurat package) to visualization relative gene expression in NPCs across all protocols.

The nephron develops from NPCs which are a heterogeneous population of mesenchyme that undergo a mesenchyme to epithelial transition (MET) in response to signals from the ureteric epithelium, giving rise to the entire nephron epithelium $[67,68]$. In vivo analysis has shown markers like SIX1, SIX2, CITED1, DAPL1 and $L Y P D 1$ are expressed in this population and can be used to reliably identify these cells from the surrounding stromal mesenchyme in situ $[17,27]$. These markers have also been used to identify the NPC populations of cells in both HFK and organoids in single-cell datasets. When analysing NPC from within the reference HFK dataset using DevKidCC, we can see that $44.9 \%$ of cells express SIX2, 56.3\% express SIX1, 53.3\% CITED1 while over 70\% express DAPL1 and LYPD1. Importantly, the kidney is the third excretory organ to arise during development. The final kidney is comprised of metanephric nephrons and is preceded by the pronephric and mesonephric tubules [69]. These arise in an anterior to posterior manner, which is reflected in their respective HOX codes. Within the HFK reference data set, the NPCs that give rise to the metanephric nephrons express a posterior HOX code, particularly the HOX10 and HOX11 paralogues [70, 71]. The posterior HOX genes are expressed, with HOXA10 most abundant and HOXC10, HOXD10, HOXA11 and $H O X D 11$ at lower levels and in less cells. The heterogeneity of gene expression within this population could result from data sparseness, dropout levels and capture bias. It may also be explained by transcriptional bursting [72], where genes are not constantly being transcribed and so the sample harvesting may occur during a transcriptional lull. However, this does provide a true reference for comparison to the expression profiles expected within these cell populations in organoids.

When we compare organoid NPCs to the HFK reference, we again note variance between publications and protocols. While the majority of organoid datasets are end-stage and thus are largely depleted of NPCs, this 
analysis confirmed previous studies showing a population of NPCs, sometimes referred to as mesenchymal progenitors, can remain. Organoids containing more than 30 NPC cells were analysed for the expression of NPC markers. Takasato protocol [63]-derived NPCs show expression of the posterior HOX code and in many samples known NPC markers, while the Morizane protocol [64], Freedman protocol [66] and Low protocol [41]-derived NPCs lack expression of posterior HOX genes, but do express some expected NPC markers, most abundantly LYPD1 and SIX1 while these are limited in Morizane protocol [64] and Freedman protocol [66]-derived NPCs, with almost no SIX2, CITED1 and DAPL1 present. Analysis of NPCs in end-stage organoids is not optimal as the prolonged culture may cause some transcriptional variability. Indeed, differences in mouse NPCs across developmental time have been characterised [73]. However, these traits are observed in samples of younger organoids and even monolayer time-points (Fig. 5A). In the 'unassigned' and 'NPC-like' populations generated in organoids, expression of the muscle markers, including MYOG and MYOD1 was sometimes evident. A subset of individual cells within such a published 'muscle' cluster [13] were re-classified by DevKidCC as NPC but do show expression of these muscle genes (Fig. 5A, Additional file 1: Figure S3). Indeed, muscle gene expression is detectable in kidney organoid clusters previously labelled as NPC from multiple protocols and publications $[12-15,31]$. However, there is no evidence for the expression of these genes in the HFK reference, suggesting that their consistent expression in organoid populations is an artefact of the in vitro culture conditions. During in vivo kidney development NPCs undergo a balance of self-renewal and commitment to nephrogenesis, allowing for ongoing waves of nephron formation leading to an average of 1 million nephrons per human kidney [74]. However, developing organoids in vitro undergo limited nephrogenesis, leading to 10 to 100s of nephrons per organoid [13]. This variation is presented grouped by experiment and timepoint, with the NPC percentage decreasing with time (Fig. 5B). The Low et al. [41] samples show an NPC peak at day 12 before a decrease by day 14 coinciding with the appearance of nephron populations. As organoids age, the NPC and NPC-like populations decrease or deplete while nephron and stromal populations increase, likely at a faster rate than is required to maintain an NPC population for ongoing nephrogenesis. In summary, we have identified an in vitro culture artefact muscle gene signature within the NPC population present across multiple protocols, giving a target to modulate for improving NPC identity within organoids. We also identify a decrease in expression of key NPC genes, including SIX2 which in mouse is believed to govern self-renewal, indicating a potential cause for the lack of ongoing nephrogenesis in vitro. This analysis demonstrates how using DevKidCC to classify and directly compare all published organoids datasets can improve our understanding of NPC population generated across multiple kidney organoid protocols.

\section{Application of DevKidCC to investigate the impact of retinoic acid on kidney organoid maturation}

Accurately identifying the cell types present within an organoid is crucial for the analysis of disease states or the optimization of the differentiation protocols. To evaluate the application of DevKidCC in analysing functional differences between methods, we analysed unpublished data in which kidney organoids from the same starting cell line generated from the same batch were treated with $5 \mu \mathrm{M}$ retinoic acid (RA) after removal of all other growth factors at day 12 of the Takasato protocol [63] to promote maturation. Mammalian nephrogenesis in vivo occurs in waves with new nephrons constantly forming up to 36 weeks gestation $[75,76]$ in humans and into the first week of life in mice [77]. This is facilitated by the presence of a peripheral nephrogenic niche within which the NPC balance self-renewal versus nephron commitment. Once differentiated, NPCs exist throughout the duration of organoid culture and deplete with time, although a population does remain in mature organoids able to undergo nephrogenesis when induced with a canonical Wnt agonist [13] (Fig. 4A). Retinoic acid signaling plays many roles in kidney development depending on spatiotemporal expression [78-80] and is also known to promote the differentiation of progenitor cell populations [81]. We investigated adding all-trans retinoic acid to organoids at multiple time points to see what effect this would have on organoids. The addition of 1-5 $\mu \mathrm{M}$ RA before day five of 3D organoid culture, substantially impaired nephron formation, whereas addition at day five onwards led to organoids with fully segmented nephrons similar to organoids without RA (data not shown). The DevKidCC classification identified an increase in the percentage of classified stromal cells, seemingly at the expense of the 'unassigned' population. In contrast to control organoids from the same batch (How_T_D25) [13] and organoid datasets of the same line, age and differentiation protocol (LVH_T_Hand_D25 and LVH_T_Dot_D25) at day 25 , the addition of RA resulted in a complete depletion of NPC cells (Fig. 6A). While the percentage of nephron cells did not change, there was a shift towards early proximal tubule (EPT) (Fig. 6B). The addition of RA also drove an increase in the presence of classified stroma at the expense of unassigned cells. These comparisons indicate that RA caused the depletion of NPCs, expansion of renal stroma and proximalisation of nephrons within forming 


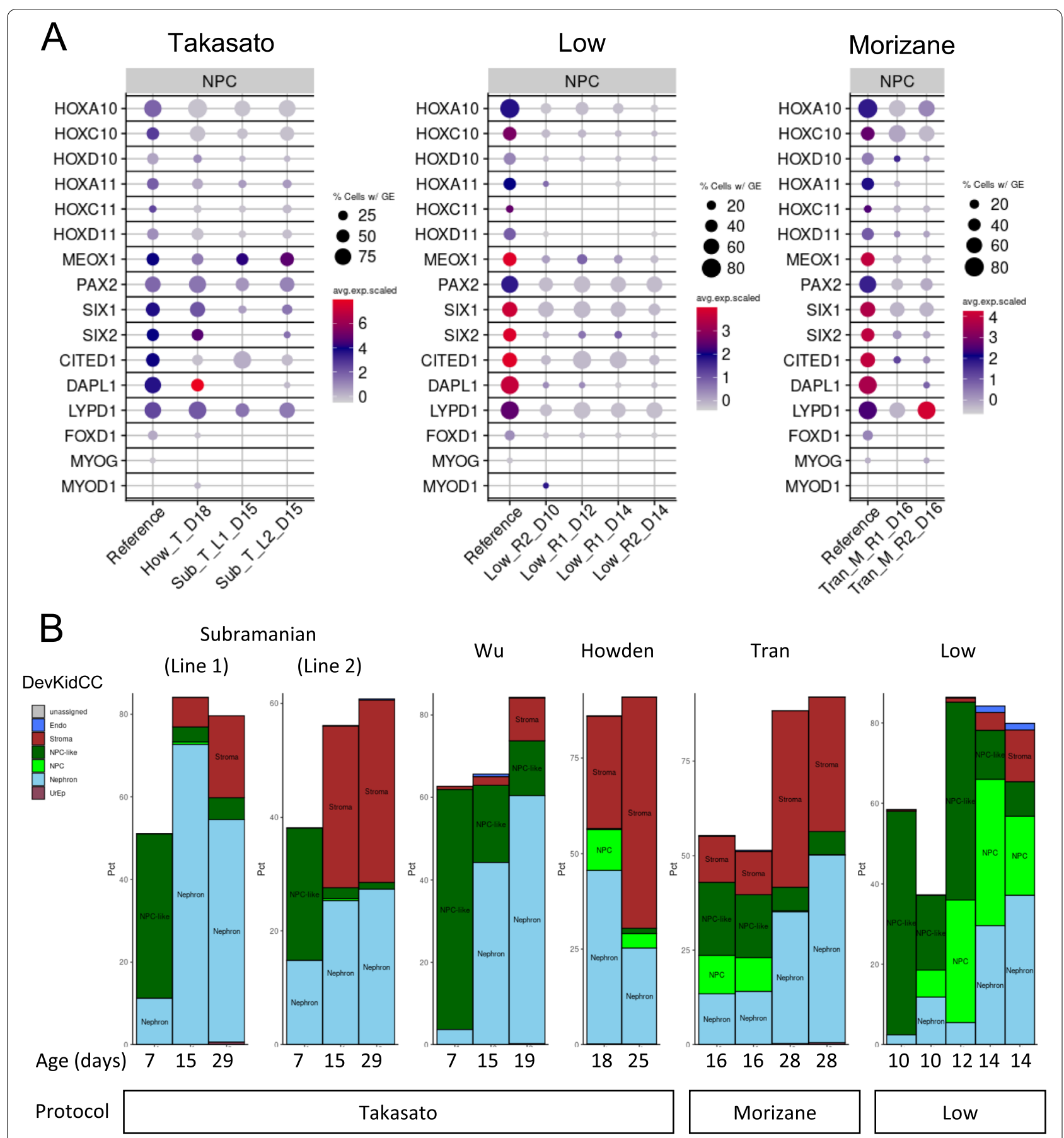

Fig. 5 Direct comparison of NPCs generated from different protocols. A Gene expression of all samples day 16 or less, grouped by protocol. B Proportions of classified cells for groups of samples with time-course information ( $y$-axes unique to each plot). NPC nephron progenitor cell

(See figure on next page.)

Fig. 6 Effect of retinoic acid when added to mid-stage organoids. A ComparePlot comparison of control and treated organoids datasets grouped by tier 1 lineage classification. B ComparePlot comparison of control and treated organoids showing the nephron identity grouped by cell sub-type. C FACS plot showing effect of RA addition on SIX2+ cell population. D Expression of PT and Pod gene markers in control and treated organoid datasets. E Expression of CLDN1 in control (left) and treated (right) organoids. RA retinoic acid, PT proximal tubule, Pod podocyte 


\section{A Tier 1 Classification}

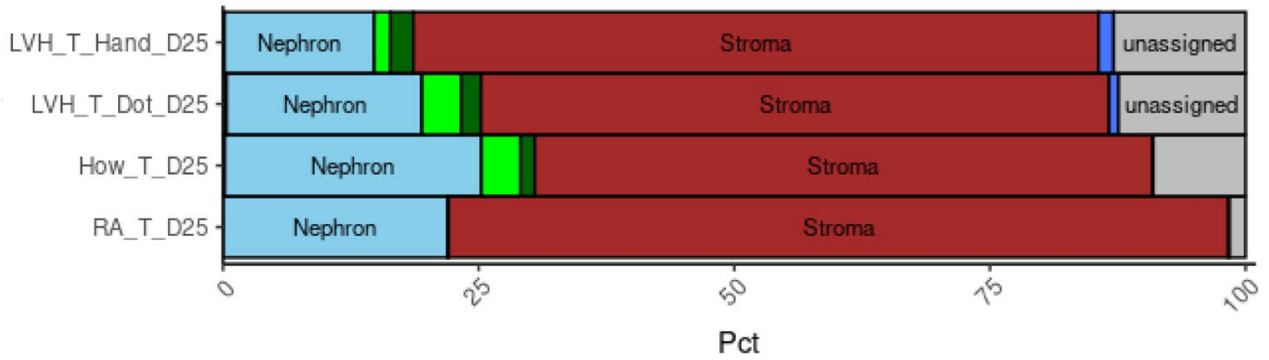

Identity

B Nephron subset classification

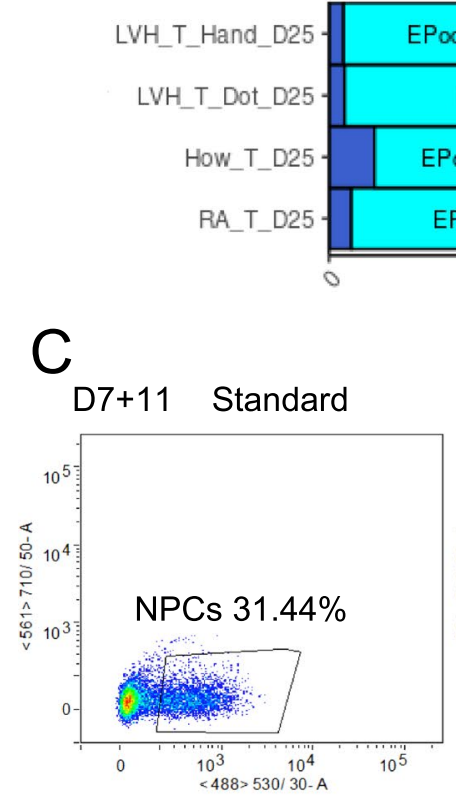

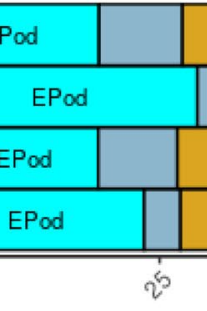

\begin{tabular}{|l|l|}
\hline EPT & \\
\hline & \\
\hline \multicolumn{2}{|c|}{ EPT } \\
\hline
\end{tabular}
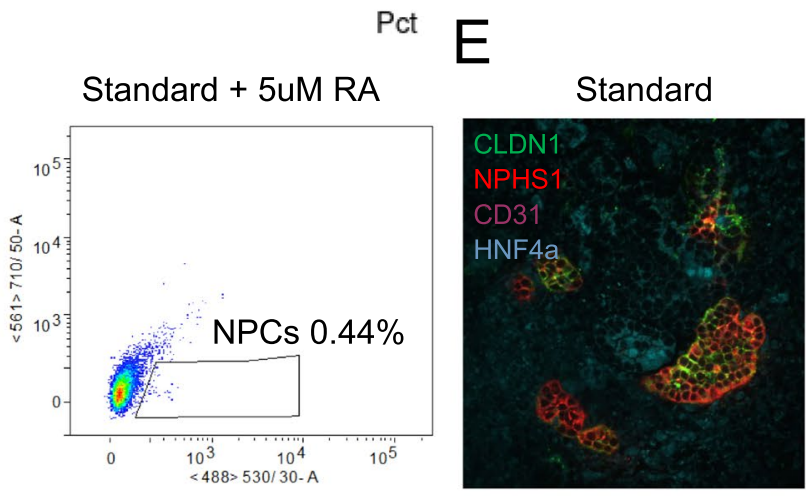

Standard + 5uM RA

D

$$
\begin{array}{r}
\text { LVH_T_Hand_D25 } \\
\text { LVH_T_Dot_D25 } \\
\text { How_T_D25 } \\
\text { RA_T_D25 } \\
\text { Reference }
\end{array}
$$

HNF4A
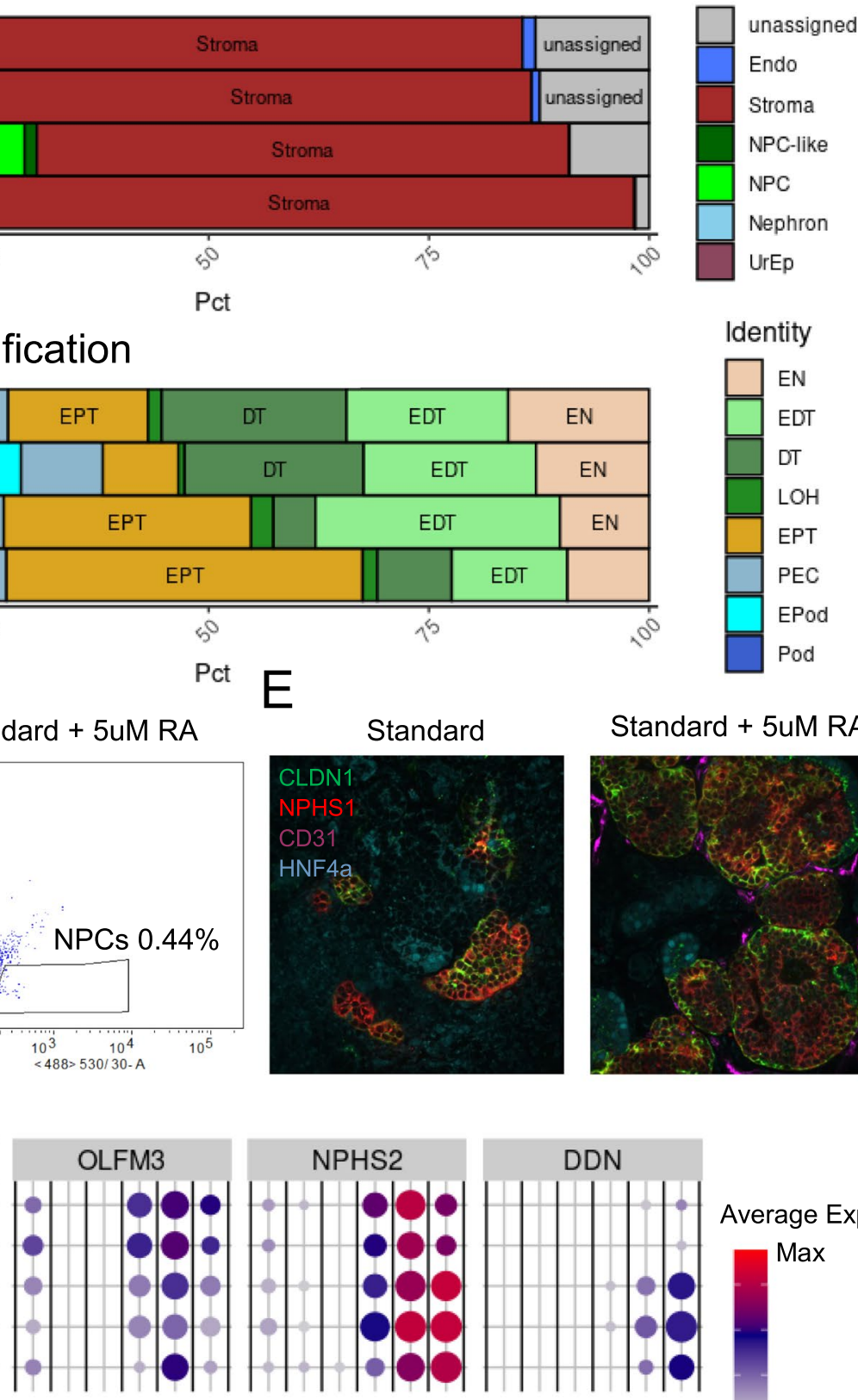

Identity
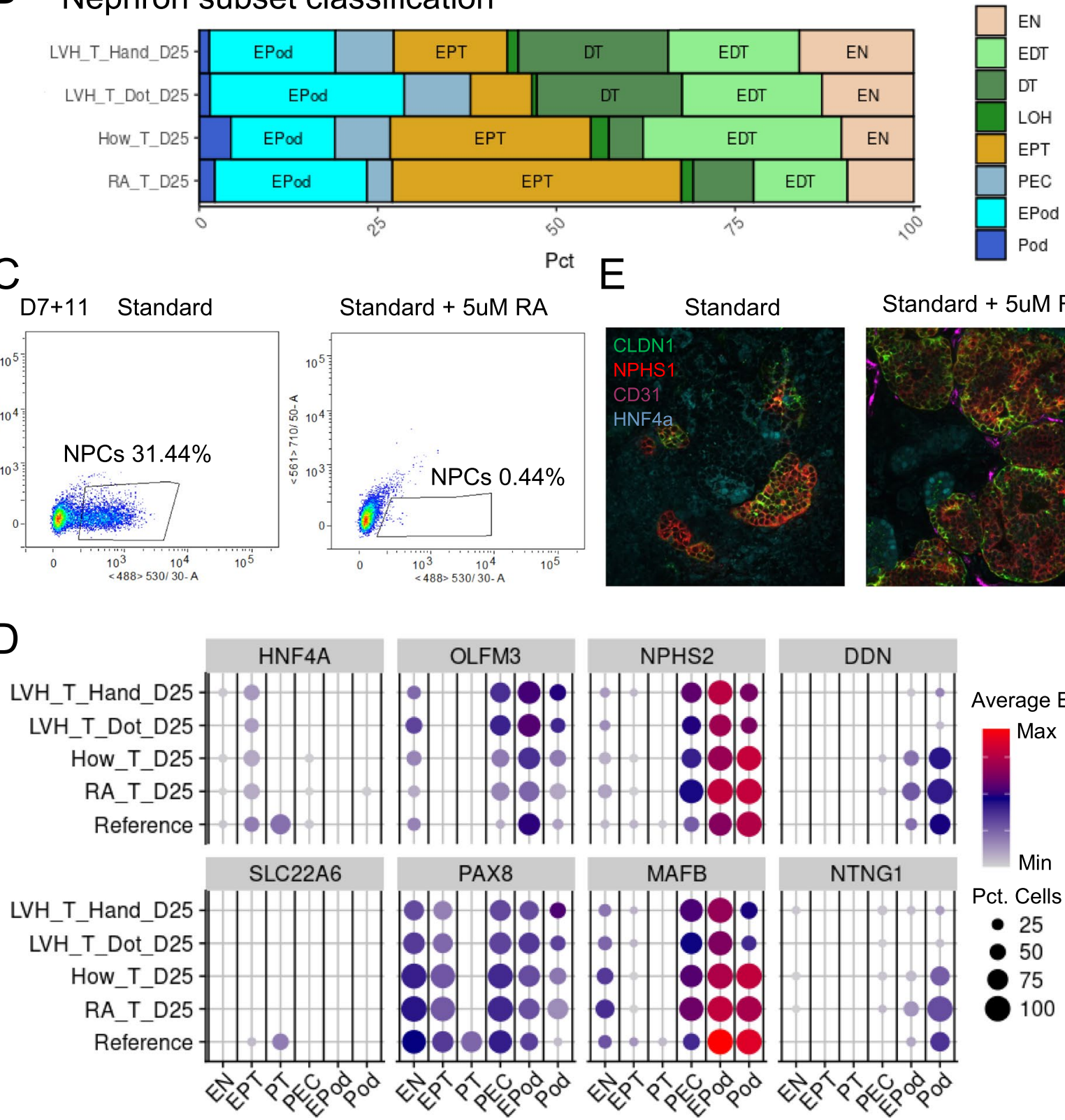

Average Exp.

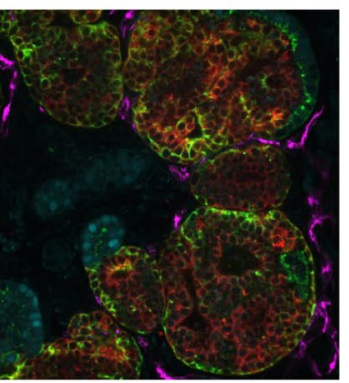

Fig. 6 (See legend on previous page.) 
organoids. To confirm if NPCs were indeed depleted by RA addition, organoids were generated using a SIX2 ${ }^{\mathrm{EGFP}}$ reporter line $[13,59]$ with and without the addition of RA and analysed using flow cytometry after 7 days. The control organoids had $31.44 \%$ EGFP+ cells while the organoids with RA had less than $0.5 \%$ (Fig. 6C). This confirms that RA acts directly or indirectly on the NPC population, forcing them to either undergo commitment to form nephrons or differentiate away from NPC identity down a stromal pathway.

To investigate the maturation of the nephrons we visualized maturation markers both proximal tubule and podocytes using the DotPlotCompare function within the package. While there was an increase in the percentage of nephron cells identified as proximal tubule, this was entirely EPT and there was no evidence of increased maturation at a transcriptional level with no expression of mature PT genes like SLC22A6. (Fig. 6D). There was an increase in the expression of podocyte maturation genes such as DDN, NTNG1 and NPHS2 with RA addition, corresponding with a decrease in OLFM3 and $P A X 8$ expression as predicted for genes expressed in immature podocytes but downregulated with maturation [24]. Immunofluorescence showed PEC marker CLDN1 had improved localization to the epithelial cells surrounding the podocytes, which is the normal location of PECs (Fig. 6E). The expression of both PEC and podocyte markers in cells assigned to all three renal corpuscle identities is consistent with the previous analysis of these populations and may indicate that the delineation of specific gene signatures within these cells is not yet occurring.

\section{Analysis of existing protocols for the development of ureteric epithelium}

The ureteric epithelium in the mammalian kidney arises as a side branch of the mesonephric duct that grows into the presumptive kidney mesenchyme [82]. Hence it has been suggested that it is not possible to generate ureteric epithelium using the same differentiation protocol able to generate the nephron lineages [83]. Single-cell analyses have recently revealed the significant transcriptional congruence between the distal nephron and the ureteric epithelium in both human and mouse [12, 16]. It has also been established that distal nephron from standard organoids remains plastic and can be induced to adopt a ureteric epithelial fate [18]. To date, a number of protocols have been published that report the generation of ureteric epithelium $[45,47,49,83,84]$ both from single monolayer differentiations generating both nephron and ureteric segments, or the isolation of cellular fractions that are then cultured separately to form ureteric epithelium. While organoids generated from a single differentiated monolayer have been reported to contain both nephron and ureteric lineages [13, 45, 63], this was due predominantly to expression of markers like GATA3 and HOXB7 which have been further identified as expressed in distal nephron segments [16, 18, 47].

As DevKidCC had shown an accurate delineation of UrEp and Distal Nephron in the HFK samples (Additional file 1: Figure S2B), we investigated the DevKidCC classification of four single cell samples claiming substantial UrEp generation using different approaches; one from a targeted UrEp differentiation [49], one from UrEp that had been derived from DN [47] and two from organoid samples generated either using the Takasato protocol or a mixed-culture approach with further UrEp-enhancing culture conditions [45] (Table 1). DevKidCC classified $32.41 \%, 21.96 \%, 1.87 \%$ and $26.81 \%$ of all sample cells as UrEp, respectively (Fig. 7A). The targeted UrEp cultures retained a more proliferative tip-like identity while the organoid cultures had a more stalk-like identity (Fig. 7B). Reflecting the methods of culture, the targeted UE cultures had nephron segments almost exclusively DN, while the organoid cultures also contained proximal segments (Fig. 7C). The absence of NPC-like cells in the DNisolated and recultured cells while their presence in the directly differentiated UrEp may be explained by the different protocols used to generate UrEp and kidney developmental biology. Cultures differentiated towards an anteriorised intermediate mesoderm population directly from hPSCs are likely to generate a proportion of NPClike cells as a bona fide posterior intermediate mesoderm of a more anterior nephrogenic cord, such as the mesonephric tubules. In contrast, the $\mathrm{DN}$-derived cultures were depleted of mesenchymal cells.

When we compare the distribution of probabilities for the nephron and UrEp populations between samples, we see a broader range of scores in both targeted cultures compared to the Uchimura organoids (Fig. 7D, E). The targeted cultures are differentiated in the absence of any supporting stromal populations, instead the signalling factors required for specifying the cell identity are added to the media. While this also occurs in the Uchimura et al. [45] organoids, they are further supported by additional mesenchymal populations as would be the case in vivo. An emerging understanding of the importance of stroma in signalling and patterning both in vivo and in vitro may provide some key as to why there is less specificity within these direct, isolated cultures $[83,85]$.

\section{Gene expression database and interactive app}

The capacity to investigate published single cell datasets is limited. The analysis provided in an original publication is generally the only output available without downloading and reanalysing a dataset. Kidney Interactive 
A Lineage classification

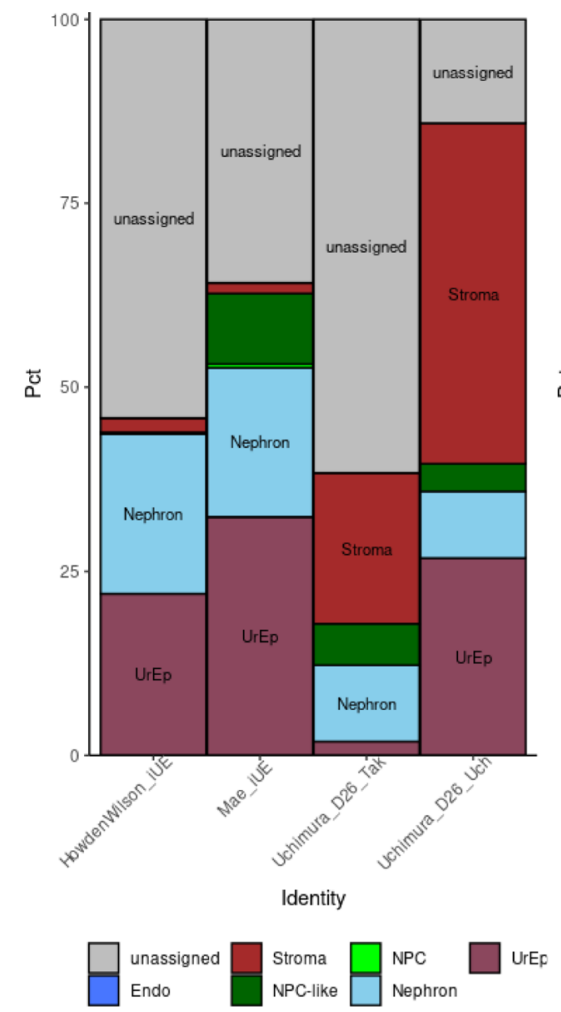

B UrEp classification

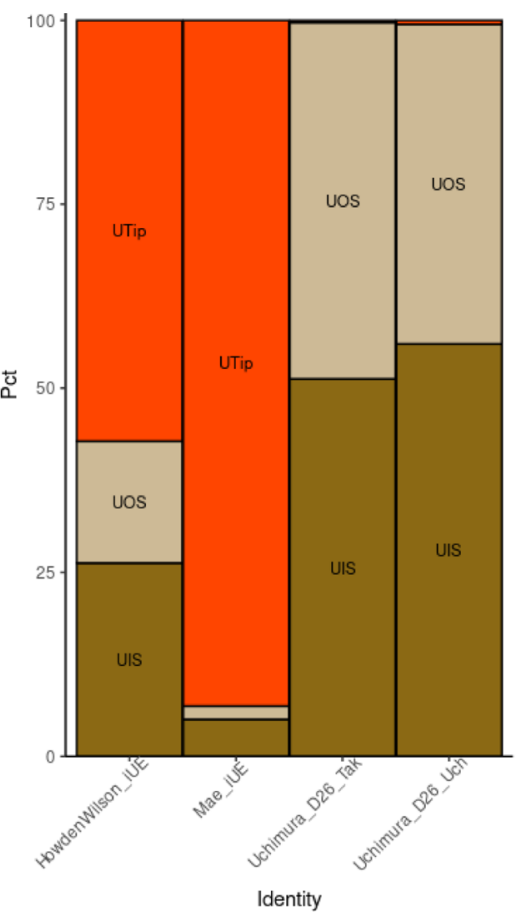

UTip $\square$ vos $\square$ UIS
C Nephron classification

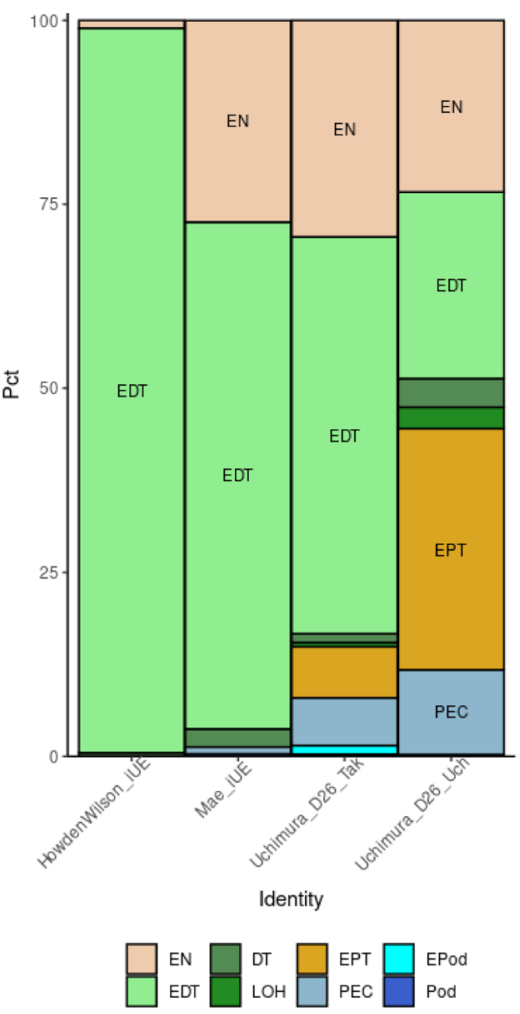

D Nephron cell probability distributions

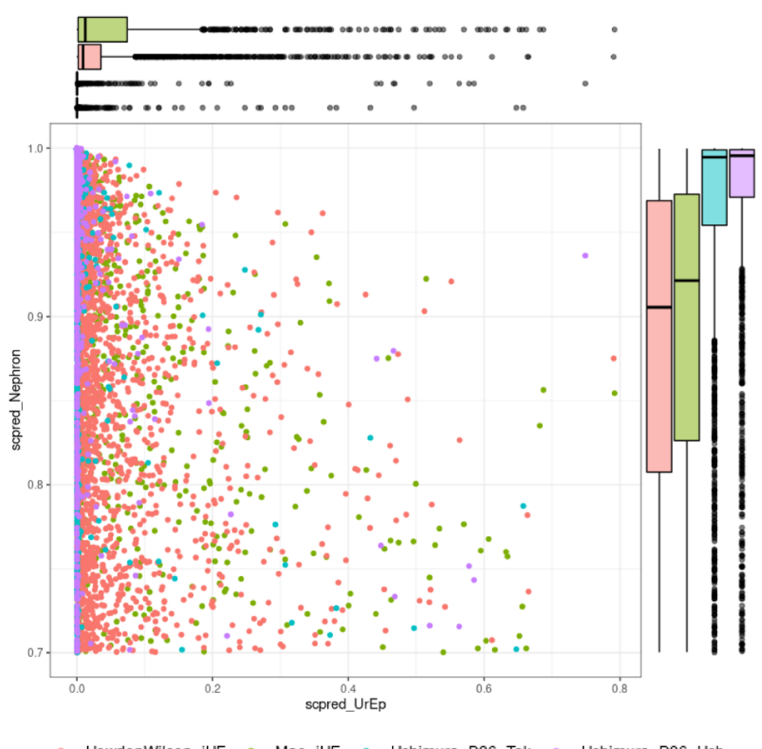

E UrEp cell probability distributions

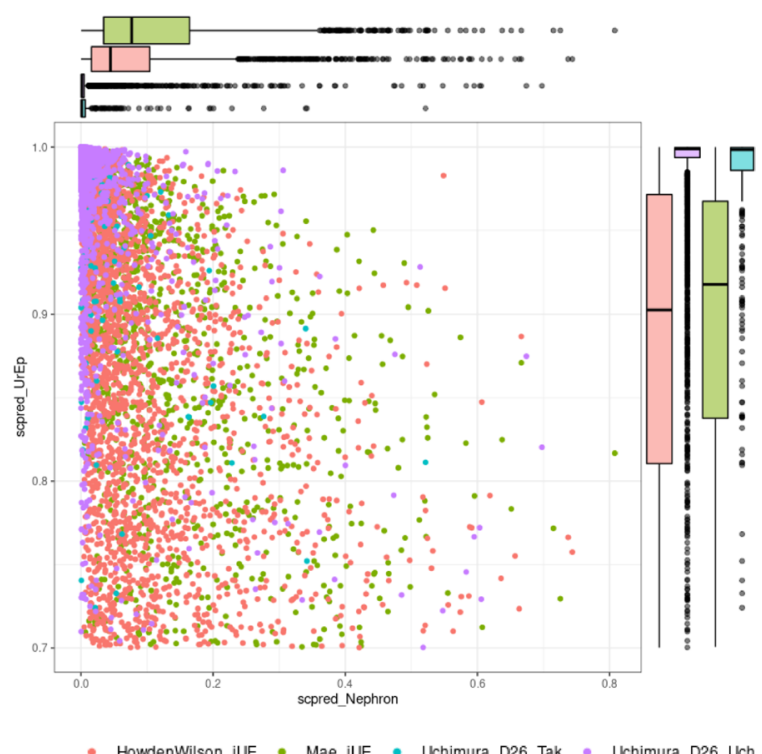

Fig. 7 Classification of ureteric cell types in organoid and targeted cultures. A The DevKidCC classification for the in vitro samples targeting UrEp culture. B Further classification of all UrEp cells from previous panel. C Further classification of all nephron cells from panel A. D Comparison of nephron and UrEp probability scores for all nephron classified cells. E Comparison of nephron and UrEp probability scores for all UrEp classified cells. UrEp ureteric epithelium 
Transcriptomics (KIT) site (http://humphreyslab.com/ SingleCell/) [86] provides analysis of a number of published HFK and organoid datasets [12, 20, 87] together with data from adult human and mouse healthy and injured kidney [86, 88, 89], allowing visualization of genes within these datasets in TSNE and Expression Plot formats. The Cello interactive app (https://cello.shinyapps. io/kidneycellexplorer/) [18] provides a visualization of gene expression broken down by carefully annotated subpopulations of the kidney nephron as expressed within adult mouse tissues. These useful tools enable the investigation of gene expression in kidney cell types; however, they do not provide a way to directly compare different datasets for relative proportions or levels of gene expression within component cells. We provide an interactive shiny app, freely available (https://kidneyregeneration. github.io/DevKidCC/articles/ShinyApp.html) [58], which allows for the investigation of gene expression in all organoid datasets as classified using DevKidCC. Using this App, gene expression can be directly compared between any included datasets, allowing for real-time investigation by all researchers. The tool provides a resource for researchers interested in gene expression and/or cell populations to identify the organoid protocol that would best suit their application, whether that be developmental biology, drug screening or clinical purposes.

\section{Discussion}

The question of cell identity is one that is difficult to answer. Histologically, we can try to define a cell type based on its morphology, gene expression or protein expression, the latter typically being read by immunohistochemistry and immunofluorescence assays. In many cellular states, particularly those present during organogenesis, evaluation of cellular identity by functional assays is challenging and marker expression is rarely unique. This challenge is significant when evaluating cell identity using single-cell RNA sequencing data. Such data is sparse, providing an incomplete snapshot rather than a comprehensive picture. As capture technology and bioinformatics tools have improved, increased levels of information can be extracted from this data, providing an overall synergy of expression profile for groups of cells within a sample. This can be combined with the pseudotime trajectory or even molecular lineage tagging to relate cells within a sample by history, assisting in likely classification of cell type. Such inferences are much more difficult in a synthetic in vitro system such as hPSCderived organoids. Such protocols direct cells to undergo a series of changes that attempt to replicate the in vivo process. However, in reality hPSC-derived lineages often do not completely recapitulate their in vivo counterparts, at least at the level of the transcriptome. We can often identify a gene, or a number of genes, expressed in a cell that provides information of its identity, but in many cases, there is ambiguity. This is compounded by our knowledge that hPSC-derived organoid models replicate early developmental cell states that are frequently in flux, not present in adult tissue and are less well defined.

The classification of cells within all single-cell data has been inconsistent as clustering and classification decisions vary between individual researchers and the limitations within each dataset. The arbitrary nature of classifying cells using clustering algorithms is challenged when identifying cells transitioning between populations, often represented as the 'borders' of clusters. The cluster-based classification of such cells will change with different approaches to analysis. The application of a cell-centred identification approach circumvents this challenge. DevKidCC represents a method of specifically classifying individual cellular identity within hPSCderived kidney organoids based predominantly upon set models trained on a comprehensive reference dataset. It should be noted however that these models are indirectly dependant on cluster-based analysis as the reference itself was initially annotated this way. Our tool facilitates direct comparisons between kidney organoid datasets by classifying cells based on the reference data. The base package, scPred [53], includes a way to integrate the data within the models using Harmony [56], although this can introduce false correlations and overcorrections between similar cell populations such as the mesenchymal cells that have intermediate to high scores for both stroma and NPC. However, batch differences are a confounding source of variation that must be taken into account. Hence, DevKidCC runs one round of harmonization using the scPred's inbuilt application of Harmony. This leads to potential iPSC-derived off-target populations with muscle or neural gene expression to be classified as NPC. This may be a result of the binary classifier for NPC not having enough information to delineate the cell types that have diverged from the NPC developmental trajectory, an example of in vitro artefacts. NPCs are an interesting population as some key markers, including CITED1, are not actually required for NPCs to become nephrons but are involved in other regulatory processes. To incorporate biological knowledge to refine the NPC classification, DevKidCC performs a further evaluation of $P A X 2$ expression to refine NPC classification. PAX2 is a known in vivo marker of nephron identity not expressed in the stroma. Indeed it has been shown to repress stromal identity [60] and is an accurate marker of nephron lineage identity in single-cell kidney datasets. The classification for all datasets has been integrated into functions allowing for plotting any novel dataset in direct comparison using the classification from DevKidCC. A suite of 
custom visualisation functions is included in DevKidCC to provide a classification and visualization toolset to investigate cell identity and gene expression within novel and existing kidney organoids.

DevKidCC was developed so that it could be applied to novel datasets facilitating direct comparisons to those previously generated. This will make comparative studies much easier, facilitating the analysis of genetic variants, disease states or methodological variation in new protocols. While this system has developed a model with three tiers of subclassification, the complexity of the human nephron, even in the fetal kidney, is such that there is scope to interrogate individual cellular identity even further within this and other subcomponents. As these models were trained using developing HFK, the ability of the tool to accurately classify cell identity during earlier stages of mesoderm patterning or mature kidney is limited. The adult kidney shows significant specification of functional cell types within all segments of the final nephron, many of which have distinct functional roles in renal filtration and fluid homeostasis but are not present in the fetal organ. Indeed, the ratio of epithelium to stroma is dramatically shifted in the adult. While the fetal kidney begins to show expression of maturing cellular states, including expression of intercalated and principal cell identities within the distal nephron/collecting duct, it is likely that a distinct cellular identity tool will be required for the accurate identification of cellular identity in postnatal kidney tissue. Conversely, the use of HFK from Trimester 1 and 2 as the reference dataset limits the ability to identify earlier stages of morphogenesis. This may explain the large percentage of unassigned cell calls in datasets in early stages of kidney organoid differentiation protocols (Fig. 4A). However, DevKidCC applied to early-stage differentiations (day 7, intermediate mesoderm) split cell identity between NPC and unassigned, suggesting that the tool is able to identify those cells beginning to commit to the mesenchymal precursors of the kidney. Indeed, in a dataset that includes day 7, 15 and 29 organoids between two cell lines [14], there is a direct relationship between the proportion of cells classified as NPC at day 7 to the proportion of nephron cells at day 15 and 29 (Fig. 5B). We conclude that at this early stage the cells identified as NPC at this early stage could be the percentage of the differentiation correctly patterned to intermediate mesoderm and are still the cells that will go on to form the nephron population.

The generation of mature nephron structures is a challenge still facing the field and is a focus of current research. It is generally accepted that current organoid protocols generate tissues transcriptionally and morphologically similar to trimester 1 and 2 stages of development. To fully utilise organoids for disease and toxicology studies, optimisation of protocols to generate mature and functionally relevant tissues is essential. Here we show how the addition of retinoic acid impacts the cellular composition of organoids by depleting NPC cells when added after the point at which nephrogenesis has begun at day 12 in Takasato organoids. This also seems to increase the percentage of classifiable renal stroma compared to off-target mesenchyme, as well as increasing the proportion of EPT compared to other nephron subtypes. While there was minimal transcriptional evidence for an increase in podocyte maturation, the improved localisation of PEC marker CLDN1 to cells surrounding the podocytes in the glomerulus would indicate there is a positive effect on glomerular maturation.

\section{Conclusions}

DevKidCC provides a robust, reproducible and computationally efficient tool for the classification of kidney single-cell data, in both human and organoid-derived tissue. Using DevKidCC, we can now directly compare between kidney samples regardless of batch and have done so for all available published datasets. This important advance has provided insights into differences in organoids derived using different protocols and allows for any novel dataset to be directly compared to all previous datasets. The included custom functions simplify visualisation of cell identity proportion and gene expression within samples and between multiple samples. Any novel dataset can be classified using the framework provided in this package, allowing for direct comparison to all previous datasets, all of which are included within the package. For visualisation of gene expression profiles and organoid cell identities, the gene expression profiles of all datasets have been built into an $R$ Shiny app available at https:// kidneyregeneration.github.io/DevKidCC/articles/Shiny App.html [58] that does not require the use of $R$ directly, allowing for easy access to this information. Finally, while this package has been built using HFK data to classify kidney cells, the framework can be transferred to any tissue type where adequate single-cell data is available.

\footnotetext{
Abbreviations

HFK: Human fetal kidney; UTip: Ureteric tip; UOS: Ureteric outer stalk; UIS: Ureteric inner stalk; SPC: Stromal progenitor cells; CS: Cortical stroma; MS: Medullary stroma; MesS: Mesangial cells; Endo: Endothelium; NPC: Nephron progenitor cell; EN: Early nephron; EDT: Early distal tubule; DT: Distal tubule; LOH: Loop of Henle; EPT: Early proximal tubule; PT: Proximal tubule; PEC: Parietal epithelial cell; EPod: Early podocytes; Pod: Podocytes; UrEp: Ureteric epithelium; DN: Distal nephron; PN: Proximal nephron; RC: Renal corpuscle; AUROC: Area under the receiver operating characteristic curve; AURPG: Area under the precision recall gain curve; SEM: Standard error of the mean; ES: Embryonic stem cell; iPS: Induced-pluripotent stem cell; hPSC: Human-derived pluripotent stem cell; MET: Mesenchyme-to-epithelial transition; RA: Retinoic acid; KIT: Kidney Interactive Transcriptomics; TSNE: t-Distributed stochastic neighbour embedding; UMAP: Uniform manifest approximation and projection.
} 


\section{Supplementary Information}

The online version contains supplementary material available at https://doi. org/10.1186/s13073-022-01023-z.

\section{Additional file 1. This file contains Figures S1 to S4. \\ Additional file 2. This file contains Table S1A-B.}

Additional file 3. This file contains Table S2.

\section{Acknowledgements}

The authors would like to thank and acknowledge constructive discussions that contributed to this manuscript development by all members of the Little laboratory and Powell laboratory.

\section{Authors' contributions}

SBW, MHL and JEP conceived the study. SBW, JAH and JEP contributed to the method development. SBW performed bioinformatics analysis. SBW, SEH, JMV and $\mathrm{AD}$ performed kidney differentiation experiments, immunofluorescence and FLOW analysis. SBW and MHL wrote the manuscript while all authors assisted in manuscript preparation. All authors read and approved the final manuscript.

\section{Funding}

This work was supported by the Australian Research Council (SR1101002: Stem Cells Australia; DP190101705, DP180101405) and the National Institutes of Health (UH3DK107344). MHL is a Senior Principal Research Fellow of the National Health and Medical Research Council, Australia (GNT1136085) and is supported by the Novo Nordisk Foundation Center for Stem Cell Medicine (NNF21CC0073729). JEP holds a National Health and Medical Research Council Investigator Grant (APP1175781).

\section{Availability of data and materials}

DevKidCC is available from Github at https://github.com/KidneyRegeneration/ DevKidCC [57]. DevKidCC Kidney Organoid Gene Expression interactive shiny dashboard is available at https://sbwilson91.shinyapps.io/devkidcc_inter active/ [58] and from Github at https://github.com/KidneyRegeneration/DevKi dCC_Interactive [58]. This software is freely available to use, copy, modify, merge, publish and distribute subject to copyright under the MIT Licence (https://github.com/KidneyRegeneration/DevKidCC/blob/main/LICENSE). Single-cell RNA-sequencing human fetal kidney datasets can be found in GEO (GSE102596, GSE1 14530) and EMBL-EBI ArrayExpress (E-MTAB-9083) [21, $23,28]$. Single-cell RNA-sequencing organoid datasets can be found in GEO (GSE118184, GSE109718, GSE1 19561, GSE1 14802, GSE115986, GSE132026, GSE124472, GSE152014, GSE161255, GSE152685, GSE131086) [25, 30, 33-35, $40,42,44,46,48]$. The single-cell RNA-sequencing organoid dataset generated in this study is available at GSE165408 [90].

\section{Declarations}

Ethics approval and consent to participate

Not applicable.

\section{Consent for publication}

Not applicable.

\section{Competing interests}

The authors declare that they have no competing interests.

\section{Author details}

${ }^{1}$ Murdoch Children's Research Institute, Flemington Rd, Parkville, Victoria, Australia. ${ }^{2}$ Department of Paediatrics, The University of Melbourne, Victoria, Parkville, Australia. ${ }^{3}$ Garvan-Weizmann Centre for Cellular Genomics, The Kinghorn Cancer Centre, Darlinghurst, New South Wales, Australia. ${ }^{4}$ UNSW Cellular Genomics Futures Institute, University of New South Wales, Sydney, New South Wales, Australia. ${ }^{5}$ Department of Anatomy and Neuroscience, The University of Melbourne, Victoria, Parkville, Australia. ${ }^{6}$ Novo Nordisk Foundation Centre for Stem Cell Medicine, Copenhagen, Denmark.
Received: 17 April 2021 Accepted: 8 February 2022

Published online: 22 February 2022

\section{References}

1. Wagner DE, Klein AM. Lineage tracing meets single-cell omics: opportunities and challenges. Nat Rev Genet. 2020;21(7):410-27 Available from: https://doi.org/10.1038/s41576-020-0223-2.

2. Gitter A. Single-cell RNA-seq pseudotime estimation algorithms. Zenodo. 2018. Available from: https://github.com/agitter/single-cell-pseudotime

3. Saelens W, Cannoodt R, Todorov H, Saeys Y. A comparison of single-cell trajectory inference methods. Nat Biotechnol. 2019;37(5):547-54 Available from: https://doi.org/10.1038/s41587-019-0071-9.

4. Zappia L, Phipson B, Oshlack A. Exploring the single-cell RNA-seq analysis landscape with the scRNA-tools database. PLOS Comput Biol. 2018;14(6):e1006245 Available from: https://doi.org/10.1371/journal.pcbi. 1006245 .

5. La Manno G, Soldatov R, Zeisel A, Braun E, Hochgerner H, Petukhov V, et al. RNA velocity of single cells. Nature. 2018;560(7719):494-8 Available from: https://doi.org/10.1038/s41586-018-0414-6.

6. Bergen V, Lange M, Peidli S, Wolf FA, Theis FJ. Generalizing RNA velocity to transient cell states through dynamical modeling. Nat Biotechnol. 2020; Available from: https://doi.org/10.1038/s41587-020-0591-3.

7. Butler A, Hoffman P, Smibert P, Papalexi E, Satija R. Integrating single-cell transcriptomic data across different conditions, technologies, and species. Nat Biotechnol. 2018;36(5):411-20 Available from: https://doi.org/10. 1038/nbt.4096.

8. Stuart T, Butler A, Hoffman P, Hafemeister C, Papalexi E, Mauck WM III, et al Comprehensive integration of single-cell data. Cell. 2019;177(7):18881902.e21 Available from: https://doi.org/10.1016/j.cell.2019.05.031.

9. Abdelaal T, Michielsen L, Cats D, Hoogduin D, Mei H, Reinders MJT, et al. A comparison of automatic cell identification methods for single-cell RNA sequencing data. Genome Biol. 2019;20(1):194 Available from: https:// pubmed.ncbi.nlm.nih.gov/31500660.

10. Kiselev VY, Yiu A, Hemberg M. scmap: projection of single-cell RNA-seq data across data sets. Nat Methods. 2018;15:359 Available from: https:// doi.org/10.1038/nmeth.4644.

11. Little $\mathrm{MH}$, Combes AN. Kidney organoids: accurate models or fortunate accidents. Genes Dev. 2019;33(19-20):1319-45 Available from: https:// doi.org/10.0.4.77/gad.329573.119.

12. Wu H, Uchimura K, Donnelly EL, Kirita Y, Morris SA, Humphreys BD. Comparative analysis and refinement of human PSC-derived kidney organoid differentiation with single-cell transcriptomics. Cell Stem Cell. 2018;23(6):869-881.e8 Available from: https://doi.org/10.0.3.248/j.stem. 2018.10.010.

13. Howden SE, Vanslambrouck JM, Wilson SB, Tan KS, Little MH. Reporterbased fate mapping in human kidney organoids confirms nephron lineage relationships and reveals synchronous nephron formation. EMBO Rep. 2019;0(0):e47483 Available from: https://doi.org/10.15252/embr. 201847483.

14. Subramanian A, Sidhom E-H, Emani M, Vernon K, Sahakian N, Zhou Y, et al. Single cell census of human kidney organoids shows reproducibility and diminished off-target cells after transplantation. Nat Commun. 2019:10(1) Available from: https://doi.org/10.0.4.14/s41467-019-13382-0.

15. Combes AN, Zappia L, Er PX, Oshlack A, Little MH. Single-cell analysis reveals congruence between kidney organoids and human fetal kidney. Genome Med. 2019;11(1) Available from: https://doi.org/10.0.4.162/ s13073-019-0615-0.

16. Combes AN, Phipson B, Lawlor KT, Dorison A, Patrick R, Zappia L, et al. Single cell analysis of the developing mouse kidney provides deeper insight into marker gene expression and ligand-receptor crosstalk. Development. 2019;146(12).

17. Lindström NO, Tran T, Guo J, Rutledge E, Parvez RK, Thornton ME et al. Conserved and divergent molecular and anatomic features of human and mouse nephron patterning. J Am Soc Nephrol. 2018:ASN.2017091036 Available from: https://doi.org/10.0.6.145/asn. 2017091036.

18. Ransick A, Lindstrom NO, Liu J, Zhu Q, Guo J-J, Alvarado GF, et al. Singlecell profiling reveals sex, lineage, and regional diversity in the mouse kidney. Dev Cell. 2019;51(3):399-413.e7. 
19. Kumar SV, Er PX, Lawlor KT, Motazedian A, Scurr M, Ghobrial I, et al. Kidney micro-organoids in suspension culture as a scalable source of human pluripotent stem cell-derived kidney cells. Development. 2019;146(5):dev172361 Available from: https://doi.org/10.0.4.218/dev. 172361.

20. Phipson B, Er PX, Combes AN, Forbes TA, Howden SE, Zappia L, et al. Evaluation of variability in human kidney organoids. Nat Methods. 2019;16(1):79-87 Available from: https://doi.org/10.0.4.14/ s41592-018-0253-2

21. Holloway EM, Spence JR, Wu JH. scRNA-seq of human fetal kidney tissue. EMBL-EBI ArrayExress. 2020. Available from: https://www.ebi.ac.uk/array express/experiments/E-MTAB-9083/

22. Holloway EM, Wu JH, Czerwinski M, Sweet CW, Wu A, Tsai Y-H, et al. Differentiation of human intestinal organoids with endogenous vascular endothelial cells. Dev Cell. 2020;54(4):516-528.e7 Available from: http:// www.sciencedirect.com/science/article/pii/S1534580720305980.

23. Hochane $M$, van den Berg PR, Fan X, Adegeest E, Bialecka M, Nieveen M, et al. Single cell RNA-sequencing of human fetal kidneys. Gene Expression Omnibus. 2019. Available from: https://www.ncbi.nlm.nih.gov/geo/ query/acc.cgi?acc $=$ GSE 114530

24. Hochane M, van den Berg PR, Fan X, Bérenger-Currias N, Adegeest E, Bialecka $M$, et al. Single-cell transcriptomics reveals gene expression dynamics of human fetal kidney development. PLOS Biol. 2019;17(2):e3000152 Available from: https://doi.org/10.1371/journal.pbio.3000152.

25. Ransick A, Tran T, Lindstrom NO, De Sena Brandine G, McMahon AP. Single Cell RNA-Seq profiling of human embryonic kidney outer and inner cortical cells and kidney organoid cells. Gene Expression Omnibus. 2019. Available from: https://www.ncbi.nlm.nih.gov/geo/query/acc.cgi?acc= GSE124472

26. Tran T, Lindstrom NO, Ransick A, De Sena BG, Guo Q, Kim AD, et al. In vivo developmental trajectories of human podocyte inform in vitro differentiation of pluripotent stem cell-derived podocytes. Dev Cell. 2019:50(1):102-116.e6.

27. Lindström NO, Guo J, Kim AD, Tran T, Guo Q, De Sena BG, et al. Conserved and divergent features of mesenchymal progenitor cell types within the cortical nephrogenic niche of the human and mouse kidney. J Am Soc Nephrol. 2018:ASN.2017080890 Available from: https://doi.org/10.0.6. 145/asn.2017080890.

28. Ransick A, Kim AD, De Sena Brandine G, Lindstrom NO, McMahon AP. Single cell RNA-Seq profiling human embryonic kidney cortex cells. Gene Expression Omnibus. 2018. Available from: https://www.ncbi.nlm.nih. gov/geo/query/acc.cgi?acc=GSE102596

29. Humphreys BD. Comparative analysis of kidney organoid and adult human kidney single cell and single nucleus transcriptomes. Gene Expression Omnibus. 2018. Available from: https://www.ncbi.nlm.nih. gov/geo/query/acc.cgi?acc=GSE118184

30. Menon R, Harder JL, Kretzler M, Otto EA, Freedman BS. Enhancing human kidney organoid differentiation from pluripotent stem cells with highthroughput automation. Gene Expression Omnibus. 2018. Available from: https://www.ncbi.nlm.nih.gov/geo/query/acc.cgi?acc=GSE109718

31. Czerniecki SM, Cruz NM, Harder JL, Menon R, Annis J, Otto EA, et al. High-throughput screening enhances kidney organoid differentiation from human pluripotent stem cells and enables automated multidimensional phenotyping. Cell Stem Cell. 2018;22(6):929-940.e4 Available from: http://10.0.3.248/j.stem.2018.04.022.

32. Menon R, Otto EA, Kokoruda A, Zhou J, Zhang Z, Yoon E, et al. Single-cell analysis of progenitor cell dynamics and lineage specification in the human fetal kidney. Development. 2018;145(16):dev164038 Available from: https://doi.org/10.0.4.218/dev.164038.

33. Howden SE, Vanslambrouck JM, Little MH, Lonsdale A, Wilson SB. Fate-mapping within human iPSC-derived kidney organoids reveals conserved mammalian nephron progenitor lineage relationships. Gene Expression Omnibus. 2019. Available from: https://www.ncbi.nlm.nih. gov/geo/query/acc.cgi?acc=GSE119561

34. Phipson B, Zappia L, Combes AN. Single cell RNA-Seq of four human kidney organoids. Gene Expression Omnibus. 2018. Available from: https:// www.ncbi.nlm.nih.gov/geo/query/acc.cgi?acc=GSE114802

35. Menon R, Harder JL, Otto EA, Kretzler M. Single-cell analysis of human kidney organoids. Gene Expression Omnibus. 2019. Available from: https:// www.ncbi.nlm.nih.gov/geo/query/acc.cgi?acc=GSE115986
36. Harder JL, Menon R, Otto EA, Zhou J, Eddy S, Wys NL, et al. Organoid single cell profiling identifies a transcriptional signature of glomerular disease. JCl insight. 2019;4(1):e122697 Available from: https://www.ncbi. nlm.nih.gov/pubmed/30626756.

37. Subramanian A. Kidney organoid reproducibility across multiple human iPSC lines and diminished off target cells after transplantation revealed by single cell transcriptomics. Gene Expression Omnibus. 2019. Available from: https://www.ncbi.nlm.nih.gov/geo/query/acc.cgi?acc=GSE136314

38. Young MD, Mitchell TJ, Vieira Braga FA, Tran MGB, Stewart BJ, Ferdinand $J R$, et al. Single-cell transcriptomes from human kidneys reveal the cellular identity of renal tumors. Science (80- ). 2018;361(6402):594-9 Available from: https://doi.org/10.0.4.102/science.aat1699.

39. Kumar SV, Lonsdale A. Kidney micro-organoids generated in suspension culture. Gene Expression Omnibus. 2019. Available from: https://www. ncbi.nlm.nih.gov/geo/query/acc.cgi?acc=GSE117211

40. Low JH, Li P, Chew EGY, Zhou B. Generating patterned kidney organoids for studying development and diseases. Gene Expression Omnibus. 2019. Available from: https://www.ncbi.nlm.nih.gov/geo/query/acc.cgi?acc= GSE132026

41. Low JH, Li P, Chew EGY, Zhou B, Suzuki K, Zhang T, et al. Generation of human PSC-derived kidney organoids with patterned nephron segments and a de novo vascular network. Cell Stem Cell. 2019;25(3):373-387.e9 Available from: http://www.sciencedirect.com/science/article/pii/S1934 590919302735.

42. Lawlor KT, Vanslambrouck JM, Little MH. Comparison manual and two types of bioprinted kidney organoids by single cell RNA-seq. Gene Expression Omnibus. 2020. Available from: https://www.ncbi.nlm.nih. gov/geo/query/acc.cgi?acc=GSE152014

43. Lawlor KT, Vanslambrouck JM, Higgins JW, Chambon A, Bishard K, Arndt $D$, et al. Cellular extrusion bioprinting improves kidney organoid reproducibility and conformation. Nat Mater. 2020; Available from: https://doi. org/10.1038/s41563-020-00853-9.

44. Humphreys BD. Human pluripotent stem cell-derived kidney organoids for modeling epithelial transport and injury. Gene Expression Omnibus. 2020. Available from: https://www.ncbi.nlm.nih.gov/geo/query/acc.cgi

45. Uchimura K, Wu H, Yoshimura Y, Humphreys BD. Human pluripotent stem cell-derived kidney organoids with improved collecting duct maturation and injury modeling. Cell Rep. 2020;33(11):108514 Available from: https:// www.sciencedirect.com/science/article/pii/S2211124720315035.

46. Wilson SB, Howden SE, Little MH. Distal nephron plasticity allows the induction of ureteric tip and stalk for the modelling of collecting duct disease. Gene Expression Omnibus. 2020. Available from: https://www. ncbi.nlm.nih.gov/geo/query/acc.cgi?acc=GSE161255

47. Howden SE, Wilson SB, Groenewegen E, Starks L, Forbes TA, Tan KS, et al. Plasticity of distal nephron epithelia from human kidney organoids enables the induction of ureteric tip and stalk. Cell Stem Cell. 2020; [cited 2021 Jan 2]; Available from: https://linkinghub.elsevier.com/retrieve/pii/ S1934590920305853.

48. Mae S-I, Ryosaka M, Sakamoto S, Matsuse K, Nozaki A, Igami M, et al. Expansion of human iPSC derived ureteric bud organoids with repeated branching potential. Gene Expression Omnibus. 2020. Available from: https://www.ncbi.nlm.nih.gov/geo/query/acc.cgi?acc=GSE152685

49. Mae S-I, Ryosaka M, Sakamoto S, Matsuse K, Nozaki A, Igami M, et al. Expansion of human iPSC-derived ureteric bud organoids with repeated branching potential. Cell Rep. 2020;32(4):107963 Available from: http:// www.sciencedirect.com/science/article/pii/S221112472030944X.

50. Choi J-H, In Kim H, Woo HG. scTyper: a comprehensive pipeline for the cell typing analysis of single-cell RNA-seq data. BMC Bioinformatics. 2020;21(1):342 Available from: https://doi.org/10.1186/ s12859-020-03700-5.

51. Yingxin Lin, Yue Cao, Hani Jieun Kim, Agus Salim, Terence P Speed, David M Lin, et al. scClassify: sample size estimation and multiscale classification of cells using single and multiple reference. Mol Syst Biol. 2020;16:e9389. https://doi.org/10.15252/msb.20199389.

52. Hao Y, Hao S, Andersen-Nissen E, Mauck WM III, Zheng S, Butler A, et al. Integrated analysis of multimodal single-cell data. Cell. 2021;184(13):3573-3587.e29 Available from: https://doi.org/10.1016/j.cell. 2021.04.048.

53. Alquicira-Hernandez J, Sathe A, Ji HP, Nguyen Q, Powell JE. scPred: accurate supervised method for cell-type classification from single-cell RNA-seq data. Genome Biol. 2019;20(1):264. 
54. John CR. MLeval: machine learning model evaluation; 2020.

55. Hafemeister C, Satija R. Normalization and variance stabilization of single-cell RNA-seq data using regularized negative binomial regression. Genome Biol. 2019;20(1):296 Available from: https://doi.org/10.1186/ s13059-019-1874-1.

56. Korsunsky I, Millard N, Fan J, Slowikowski K, Zhang F, Wei K, et al. Fast, sensitive and accurate integration of single-cell data with Harmony. Nat Methods. 2019;16(12):1289-96 Available from: https://doi.org/10.1038/ s41592-019-0619-0.

57. Wilson SB, Little MH. DevKidCC: developing kidney cell classifier. Github. 2021. Available from: https://github.com/KidneyRegeneration/DevKidCC

58. Wilson SB, Little MH. DevKidCC kidney organoid gene expression shiny application. Shiny App. 2021. Available from: https://sbwilson91.shiny apps.io/devkidcc_interactive/

59. Vanslambrouck JM, Wilson SB, Tan KS, Soo JY-C, Scurr M, Spijker HS, et al. A Toolbox to characterize human induced pluripotent stem cell-derived kidney cell types and organoids. J Am Soc Nephrol. 2019;30(10):1811-23.

60. Naiman N, Fujioka K, Fujino M, Valerius MT, Potter SS, McMahon AP, et al. Repression of interstitial identity in nephron progenitor cells by Pax2 establishes the nephron-interstitium boundary during kidney development. Dev Cell. 2017;41(4):349-365.e3 [cited 2018 May 9]. Available from: https://www.sciencedirect.com/science/article/pii/S1534580717303489? via\%3Dihub.

61. Bouchard M, Souabni A, Mandler M, Neubüser A, Busslinger M. Nephric lineage specification by Pax2 and Pax8. Genes Dev. 2002;16(22):2958-70 Available from: https://pubmed.ncbi.nlm.nih.gov/12435636.

62. Kaku Y, Taguchi A, Tanigawa S, Haque F, Sakuma T, Yamamoto T, et al. PAX2 is dispensable for in vitro nephron formation from human induced pluripotent stem cells. Sci Rep. 2017;7(1):4554 Available from: https:// pubmed.ncbi.nlm.nih.gov/28674456.

63. Takasato M, Er PX, Chiu HS, Maier B, Baillie GJ, Ferguson C, et al. Kidney organoids from human iPS cells contain multiple lineages and model human nephrogenesis. Nature. 2015;526(7574):564-8.

64. Morizane R, Lam AQ, Freedman BS, Kishi S, Valerius MT, Bonventre JV. Nephron organoids derived from human pluripotent stem cells model kidney development and injury. Nat Biotechnol. 2015;33:1193 Available from: https://doi.org/10.1038/nbt.3392.

65. Tran HTN, Ang KS, Chevrier M, Zhang X, Lee NYS, Goh M, et al. A benchmark of batch-effect correction methods for single-cell RNA sequencing data. Genome Biol. 2020;21(1):12 Available from: https://doi.org/10.1186/ s13059-019-1850-9.

66. Freedman BS, Brooks CR, Lam AQ, Fu H, Morizane R, Agrawal V, et al. Modelling kidney disease with CRISPR-mutant kidney organoids derived from human pluripotent epiblast spheroids. Nat Commun. 2015;6:8715 Available from: https://doi.org/10.1038/ncomms9715.

67. Kobayashi A, Valerius MT, Mugford JW, Carroll TJ, Self M, Oliver G, et al. Six2 defines and regulates a multipotent self-renewing nephron progenitor population throughout mammalian kidney development. Cell Stem Cell. 2008;3(2):169-81 [cited 2018 May 8]. Available from: https://www.scien cedirect.com/science/article/pii/S1934590908003470?via\%3Dihub.

68. Self M, Lagutin OV, Bowling B, Hendrix J, Cai Y, Dressler GR, et al. Six2 is required for suppression of nephrogenesis and progenitor renewal in the developing kidney. EMBO J. 2006;25(21):5214-28 Available from: https:// www.ncbi.nlm.nih.gov/pubmed/17036046.

69. Saxén L, Sariola H. Early organogenesis of the kidney. Pediatr Nephrol. 1987;1(3):385-92 Available from: https://doi.org/10.1007/BF00849241.

70. Wellik DM, Hawkes PJ, Capecchi MR. Hox 11 paralogous genes are essential for metanephric kidney induction. Genes Dev. 2002;16(11):1423-32.

71. Yallowitz AR, Hrycaj SM, Short KM, Smyth IM, Wellik DM. Hox10 genes function in kidney development in the differentiation and integration of the cortical stroma. PLoS One. 2011;6(8):e23410 Available from: https:// www.ncbi.nlm.nih.gov/pubmed/21858105.

72. Chubb JR, TrcekT, Shenoy SM, Singer RH. Transcriptional pulsing of a developmental gene. Curr Biol. 2006;16(10):1018-25.

73. Chen S, Brunskill EW, Potter SS, Dexheimer PJ, Salomonis N, Aronow BJ, et al. Intrinsic age-dependent changes and cell-cell contacts regulate nephron progenitor lifespan. Dev Cell. 2015;35(1):49-62 Available from: https://www.sciencedirect.com/science/article/pii/S1534580715005894.

74. Hughson M, Farris AB, Douglas-Denton R, Hoy WE, Bertram JF. Glomerular number and size in autopsy kidneys: the relationship to birth weight. Kidney Int. 2003;63(6):2113-22.
75. Hartman HA, Lai HL, Patterson LT. Cessation of renal morphogenesis in mice. Dev Biol. 2007;310(2):379-87 Available from: https://pubmed.ncbi. nlm.nih.gov/17826763.

76. Rumballe BA, Georgas KM, Combes AN, Ju AL, Gilbert T, Little MH. Nephron formation adopts a novel spatial topology at cessation of nephrogenesis. Dev Biol. 2011;360(1):110-22 Available from: https:// pubmed.ncbi.nlm.nih.gov/21963425.

77. Short KM, Combes AN, Lefevre J, Ju AL, Georgas KM, Lamberton T, et al. Global quantification of tissue dynamics in the developing mouse kidney. Dev Cell. 2014;29(2):188-202 Available from: http://www.sciencedirect. com/science/article/pii/S1534580714001336.

78. Burrow CR. Retinoids and renal development. Nephron Exp Nephrol [Internet]. 2000;8(4-5):219-25 Available from: https://www.karger.com/ DOI/10.1159/000020672.

79. Janesick A, Tang W, Shioda T, Blumberg B. RARY is required for mesodermal gene expression prior to gastrulation. Development. 2018:dev.147769 Available from: https://doi.org/10.0.4.218/dev.147769.

80. Janesick A, Nguyen TT, Aisaki K, Igarashi K, Kitajima S, Chandraratna RA, Kanno J, Blumberg B. Active repression by RARY signaling is required for vertebrate axial elongation. Development. 2014;141(11):2260-70. https:// doi.org/10.1242/dev.103705.

81. Gudas L, Wagner JA. Retinoids regulate stem cell differentiation. J Cell Physiol. 2011;226(2):322-30 Available from: https://pubmed.ncbi.nlm.nih. gov/20836077.

82. Costantini F, Kopan R. Patterning a complex organ: branching morphogenesis and nephron segmentation in kidney development. Dev Cell. 2010;18(5):698-712 Available from: https://doi.org/10.0.3.248/j.devcel. 2010.04.008.

83. Taguchi A, Nishinakamura R. Higher-Order kidney organogenesis from pluripotent stem cells. Cell Stem Cell. 2017;21(6):730-746.e6 Available from: https://doi.org/10.1016/j.stem.2017.10.011.

84. Xia Y, Nivet E, Sancho-Martinez I, Gallegos T, Suzuki K, Okamura D, et al. Directed differentiation of human pluripotent cells to ureteric bud kidney progenitor-like cells. Nat Cell Biol. 2013;15(12):1507-15 Available from: https://doi.org/10.1038/ncb2872.

85. Wilson SB, Little MH. The origin and role of the renal stroma. Development. 2021;148(19) Available from: https://doi.org/10.1242/dev.199886.

86. Wu H, Malone AF, Donnelly EL, Kirita Y, Uchimura K, Ramakrishnan SM, et al. Single-cell transcriptomics of a human kidney allograft biopsy specimen defines a diverse inflammatory response. J Am Soc Nephrol. 2018;29(8):2069 LP-2080 Available from: http://jasn.asnjournals.org/conte nt/29/8/2069.abstract.

87. Lindström NO, De Sena BG, Tran T, Ransick A, Suh G, Guo J, et al. Progressive recruitment of mesenchymal progenitors reveals a time-dependent process of cell fate acquisition in mouse and human nephrogenesis. Dev Cell. 2018;45(5):651-660.e4 Available from: https://doi.org/10.0.3.248/j. devcel.2018.05.010

88. Wilson PC, Wu H, Kirita Y, Uchimura K, Ledru N, Rennke HG, et al. The single-cell transcriptomic landscape of early human diabetic nephropathy. Proc Natl Acad Sci. 2019;116(39):19619 LP-625 Available from: http:// www.pnas.org/content/116/39/19619.abstract.

89. Muto, Y., Wilson, P.C., Ledru, N. et al. Single cell transcriptional and chromatin accessibility profiling redefine cellular heterogeneity in the adult human kidney. Nat Commun. 2021;12:2190. https://doi.org/10.1038/ s41467-021-22368-w.

90. Wilson SB, Vanslambrouck JM, Howden SE, Little MH. Addition of retinoic acid to kidney organoids. Gene Expression Omnibus. 2022. Available from: https://www.ncbi.nlm.nih.gov/geo/query/acc.cgi?acc=GSE165408

\section{Publisher's Note}

Springer Nature remains neutral with regard to jurisdictional claims in published maps and institutional affiliations. 